\title{
Semantic variables both help and hinder word production: \\ Behavioural evidence from picture naming
}

\author{
Leonie F. Lampe ${ }^{1,2}$, Solène Hameau ${ }^{1,3}$, and Lyndsey Nickels ${ }^{1}$ \\ ${ }^{1}$ Department of Cognitive Science, Macquarie University \\ ${ }^{2}$ International Doctorate for Experimental Approaches to Language and Brain (IDEALAB), Universities \\ of Groningen (NL), Potsdam (DE), Newcastle (UK), and Macquarie University (AU) \\ ${ }^{3}$ Department of Linguistics, Macquarie University
}

\begin{abstract}
Author Note
Leonie F. Lampe https://orcid.org/0000-0001-6020-8506

Solène Hameau https://orcid.org/0000-0001-5282-2645

Lyndsey Nickels https://orcid.org/0000-0002-0311-3524

We have no known conflict of interest to disclose.

This study was preregistered on the Open Science Framework. The preregistration, data, and scripts for the analyses are available under: https://osf.io/yw6ma/

Correspondence concerning this article should be addressed to Leonie F. Lampe, Department of Cognitive Science, Macquarie University, 16 University Ave, NSW 2109, Australia.

Email: leonie.lampe@students.mq.edu.au
\end{abstract}

Funding: This research was supported by an International Macquarie University Research Excellence Scholarship (iMQRES) grant to Leonie F. Lampe. Solène Hameau was supported by an Australian Research Council Discovery Project Grant (number DP190101490) to Lyndsey Nickels.

Acknowledgements: We thank Nora Fieder and Audrey Bürki for helpful discussions when planning the experiment and Audrey Bürki for her comments on a previous version of this paper. We also thank Serje Robidoux for statistical advice and Andrew Etchell for his help when programming the experiment. Moreover, we would like to thank the JEP:LMC Editor, Prof Keith A. Hutchison, and two anonymous reviewers for helpful comments on an earlier version of this manuscript. 


\begin{abstract}
This research investigated how word production is influenced by six feature-based semantic variables (number of semantic features, intercorrelational density, number of near semantic neighbours, semantic similarity, typicality, and distinctiveness). We simultaneously investigated effects of the six semantic variables on spoken picture naming in a large group of participants $(n=87)$, while controlling for other psycholinguistic variables. Across analyses, number of semantic features was the most consistent predictor with a facilitatory effect on naming latency and accuracy. In addition, inhibitory effects were found on naming accuracy for intercorrelational density and on naming latency for distinctiveness. The facilitatory effect of number of semantic features is suggested to stem from stronger semantic activation with an increasing number of semantic features, which results in facilitated selection of the word's lexical representation. In contrast, the inhibitory effect of intercorrelational density is most easily accounted for by increased competition at the lexical level. The mechanism underlying the inhibitory effect of distinctiveness is unclear. These findings indicate that future research on factors affecting word retrieval should also control for effects of number of semantic features, intercorrelational density, and distinctiveness. They also suggest that effects of the other semantic variables (e.g., semantic neighbours) reported in the literature were potentially overestimated due to insufficient control of other semantic and/or psycholinguistic variables.
\end{abstract}

Keywords: picture naming, semantic variables, lexical selection, competition, replication 


\section{Introduction}

Decades of research targeted at understanding processes during word production have led to several theoretical accounts of word production (e.g., Abdel Rahman \& Melinger, 2009, 2019; Dell, 1986; Howard et al., 2006; Levelt et al., 1999), which mostly agree about the general architecture of word production and there is a consensus that it is a semantically mediated process (e.g., Bock $\&$ Levelt, 1994; Vitkovitch \& Humphreys, 1991; but see Kremin, 1986). The most common experimental paradigm for these investigations is picture naming. In this paradigm, following picture processing, the activation of word meaning at a semantic level leads to lexical level activation, where the target word's lexical representation is selected among (possibly competing) alternatives. This is followed by activation of the target's word form and then by further phonological and phonetic processes that lead to word production. However, there is disagreement about crucial details of the model. More specifically, it is, for example, still unclear how exactly information is processed at each level of the model and researchers disagree about whether there is competition between co-activated representations, the mechanism underlying any such competition, and the level(s) it might affect. Word production processes are often investigated by manipulating the context in which a target word appears (Picture Word Interference, e.g., Gauvin et al., 2018; Melinger \& Rahman, 2013; Blocked-Cyclic Naming, e.g., Ewald et al., 2012; Python et al., 2018; Continuous Naming, e.g., Howard et al., 2006; Rose \& Abdel Rahman, 2016). Rather than manipulating the context in which words occur, another approach to the investigation of processes in word production is to examine the effects of item-inherent characteristics that naturally differ from word to word (e.g., word frequency or imageability, e.g., Alario et al., 2004). The principle is that information processing at the different levels of word production may be affected by manipulations of, or naturally occurring variability in, these word characteristics. Hence, the effects of these word characteristics can in turn inform and advance our understanding of representation and processing in word production. One set of variables that attracts research attention are those that capture information about the semantic representation of a target word and its relationship to other words in the mental lexicon (e.g., Fieder et al., 2019; Rabovsky 
et al., 2016, 2020): semantic variables. Semantic variables operationalise statistical or distributional facts about the presumed semantic representation of a target word and can capture a wide range of aspects of the meaning of single concepts or of its relationships to other words in the mental lexicon. They can, for example, describe the richness of the semantic representation of the target word or the number of words that are closely related in meaning. It is the effects of these semantic variables on spoken word production that are the focus of the research presented here.

Most of the previous research into effects of semantic variables was conducted to investigate the underlying semantic representations themselves and thus focused on word comprehension, using tasks like semantic categorisation and feature verification, or conducted computational modelling from this perspective (e.g., Fujihara et al., 1998; McRae et al., 1997; Mirman \& Magnuson, 2008; Pexman et al., 2003; Randall et al., 2004). However, in word production, semantic processing precedes and therefore determines lexical processing. Consequently, semantic variables are hypothesised to not only have consequences for semantic processing but may also affect subsequent lexical processing. However, current theories of word production are underspecified with respect to effects of semantic variables and details of how they might affect processing are not clearly stated. This makes word production a particularly interesting and relevant modality in which to study effects of semantic variables.

Given the possibility of widespread influence of semantic variables on the word production process, it is important to establish which semantic variables reliably affect word production (as measured in picture naming) and to understand the mechanisms underlying these effects. Understanding the influence of these semantic variables will facilitate our understanding of how information is represented and processed at the different stages of word production and help us to better understand word production mechanisms. More specifically, studying semantic variables will shed light on the structure of semantic representations and their influence on retrieval and selection of words in the mental lexicon. This also allows us to evaluate the adequacy of current theories of word production, given that some theories may not include the architectural elements required to 
explain the effects observed. Moreover, knowledge of the semantic variables that influence word production is also of methodological importance, as it can, for example, be used to achieve improved matching and control of influential variables.

Depending on the theory of semantic representation, that is, the nature of the semantic dimensions encoded in the human mind, various relationships are suggested to be important, each of which represents a different approach to specifying aspects of semantic representation. Semantic relationships can be described on the basis of participant-generated associations (e.g., De Deyne et al., 2019; Nelson et al., 2004), co-occurrence patterns in text corpora (e.g., Latent Semantic Analysis, Landauer et al., 1998; Continuous bag-of-words model, Mikolov et al., 2013), or participant-generated semantic features (e.g., Devereux et al., 2014; McRae et al., 2005; Vinson \& Vigliocco, 2008).

In this study, we focus on effects of semantic variables that can be operationalised based on semantic features, as provided in the feature norm database by McRae et al. (2005). Importantly, we believe that the use of feature-based semantic variables is not only compatible with semantic knowledge being decomposed into semantic features. In contrast, feature-based semantic variables could also be indicative of relations between holistic lexical concepts like, for example, the number or strength of connections between them (e.g., Abdel Rahman \& Melinger, 2009; Collins \& Loftus, 1975; Levelt et al., 1999). In our theoretical interpretations of the findings, we use a cascading, interactive model of word production that assumes lexical competition (Abdel Rahman \& Melinger, 2009, 2019), however, we also assess the ability of other current theories of word production to account for the observed effects of semantic variables and consider both feature-based and holistic semantic representations.

Previous research into effects of semantic variables was often inconclusive and subject to methodological flaws (discussed below). Consequently, here, we aimed to extend and improve the evidence base, by investigating the effects of these semantic variables on the word production process. 


\section{Review of the previous literature on feature-based semantic variables}

Effects of semantic variables that can be operationalised based on semantic features were studied to varying degrees in previous research. Table 1 summarises effects of feature-based semantic variables from studies that used simple picture naming paradigms with neurotypical adults, and, as is apparent, both facilitatory and inhibitory effects have been observed. While facilitation in word production is usually attributed to spreading activation at the semantic level (e.g., Abdel Rahman \& Melinger, 2009) or feedback from the lexical to the semantic level (e.g., Dell et al., 1986), inhibition has been linked to competitive processes at the lexical (Abdel Rahman \& Melinger, 2009, 2019; Howard et al., 2006; Levelt et al., 1999) or post-lexical stages (Mahon et al., 2007), or to a non-competitive learning mechanism influencing the strength of connections between semantic and lexical units (Oppenheim et al., 2010). We now address each of these variables in turn. 


\section{Table 1}

Effects of semantic variables on picture naming in neurotypical adults: previous research

\begin{tabular}{|c|c|c|c|c|c|c|c|c|}
\hline Study & $\begin{array}{l}\text { Participants } \\
(n)\end{array}$ & $\begin{array}{l}\text { Items } \\
(n)\end{array}$ & Design/analyses & Operationalisation & RT & Accuracy & Semantic errors & Omissions \\
\hline \multicolumn{9}{|c|}{ Number of semantic features } \\
\hline $\begin{array}{l}\text { Rabovsky } \\
\text { et al., } 2016\end{array}$ & 16 & 541 & continuous, (g)Imer & $\begin{array}{l}\text { feature-norm database (McRae et al., } \\
\text { 2005) }\end{array}$ & $\nearrow$ & $\nearrow$ & & \\
\hline $\begin{array}{l}\text { Rabovsky } \\
\text { et al., } 2020\end{array}$ & 31 & 345 & $\begin{array}{l}\text { continuous, Imer, } \\
\text { Bayesian model }\end{array}$ & $\begin{array}{l}\text { feature-norm database (McRae et al., } \\
\text { 2005) }\end{array}$ & $\nearrow$ & $\nearrow$ & & \\
\hline $\begin{array}{l}\text { Taylor et al., } \\
2012\end{array}$ & 20 & 302 & continuous, (g)Imer & $\begin{array}{l}\text { feature-norm database (McRae et al., } \\
\text { 2005) }\end{array}$ & $\nearrow$ & & & \\
\hline \multicolumn{9}{|c|}{ Intercorrelational density } \\
\hline $\begin{array}{l}\text { Rabovsky } \\
\text { et al., } 2016\end{array}$ & 16 & 541 & continuous, (g)Imer & $\begin{array}{l}\text { feature-norm database (McRae et al., } \\
\text { 2005) }\end{array}$ & $\swarrow$ & $\swarrow$ & & \\
\hline $\begin{array}{l}\text { Rabovsky } \\
\text { et al., } 2020\end{array}$ & 31 & 345 & $\begin{array}{l}\text { continuous, Imer, } \\
\text { Bayesian model }\end{array}$ & $\begin{array}{l}\text { feature-norm database (McRae et al., } \\
\text { 2005) }\end{array}$ & $\varnothing^{a}$ & $\swarrow$ & & \\
\hline $\begin{array}{l}\text { Taylor et al., } \\
2012\end{array}$ & 20 & 302 & continuous, (g)Imer & $\begin{array}{l}\text { feature-norm database (McRae et al., } \\
2005)^{\text {b }}\end{array}$ & $\varnothing$ & & & \\
\hline \multicolumn{9}{|c|}{ Number of near semantic neighbours } \\
\hline $\begin{array}{l}\text { Fieder et } \\
\text { al., } 2019^{c}\end{array}$ & 30 & 180 & continuous, (g)Imer & $\begin{array}{l}\text { feature-norm database (Devereux et al., } \\
\text { 2014) }\end{array}$ & $\swarrow$ & $\swarrow$ & $\begin{array}{c}\nearrow \text { (also for } \\
\text { coordinate errors) }\end{array}$ & $\nearrow$ \\
\hline $\begin{array}{l}\text { Mirman, } \\
2011^{c}\end{array}$ & $\begin{array}{l}35 \text { older } \\
\text { adults }\end{array}$ & 57 & matched sets & $\begin{array}{l}\text { feature-norm database (McRae et al., } \\
\text { 2005) }\end{array}$ & $\varnothing$ & $\swarrow$ & $\nearrow$ & \\
\hline $\begin{array}{l}\text { Hameau et } \\
\text { al., } 2019\end{array}$ & 40 & 84 & continuous, (g)Imer & $\begin{array}{l}\text { feature-norm database (McRae et al., } \\
\text { 2005) and rating; feature-based } \\
\text { semantic neighbourhood factor: number } \\
\text { of near semantic neighbours and rated }\end{array}$ & $\varnothing^{d}$ & $\varnothing^{d}$ & & \\
\hline
\end{tabular}




\begin{tabular}{|c|c|c|c|c|c|c|c|c|}
\hline Study & $\begin{array}{l}\text { Participants } \\
(n)\end{array}$ & $\begin{array}{l}\text { Items } \\
(n)\end{array}$ & Design/analyses & Operationalisation & RT & Accuracy & Semantic errors & Omissions \\
\hline & & & & competitors & & & & \\
\hline $\begin{array}{l}\text { Lampe et } \\
\text { al., } 2017\end{array}$ & $\begin{array}{l}15 \text { older } \\
\text { adults }\end{array}$ & 44 & matched sets & $\begin{array}{l}\text { feature-norm database (McRae et al., } \\
\text { 2005) }\end{array}$ & $\varnothing$ & $\varnothing$ & & \\
\hline $\begin{array}{l}\text { Bormann, } \\
2011\end{array}$ & 18 & 54 & $\begin{array}{l}\text { matched sets, } \\
\text { (g)Imer }\end{array}$ & rated within category competitors ${ }^{e}$ & $\varnothing$ & & & \\
\hline \multicolumn{9}{|c|}{ Semantic similarity } \\
\hline $\begin{array}{l}\text { Fieder et } \\
\text { al., } 2019^{c}\end{array}$ & 30 & 180 & continuous, (g)Imer & $\begin{array}{l}\text { feature-norm database (Devereux et al., } \\
\text { 2014) }\end{array}$ & $\varnothing$ & $\swarrow$ & $\nearrow$ & $\nearrow$ \\
\hline \multicolumn{9}{|c|}{ Typicality } \\
\hline $\begin{array}{l}\text { Dell'Acqua } \\
\text { et al., } 2000\end{array}$ & 84 & 266 & $\begin{array}{l}\text { continuous, } \\
\text { multiple regression }\end{array}$ & rating & $\nearrow$ & & & \\
\hline $\begin{array}{l}\text { Grossman } \\
\text { et al., } 1998\end{array}$ & $\begin{array}{l}14 \text { older } \\
\text { adults }\end{array}$ & 72 & matched sets & rating & $\nearrow$ & & & \\
\hline $\begin{array}{l}\text { Holmes \& } \\
\text { Ellis, } 2006^{f}\end{array}$ & 25 & 84 & matched sets & rating & $\nearrow$ & $\varnothing$ & & \\
\hline $\begin{array}{l}\text { Jolicoeur et } \\
\text { al., } 1984\end{array}$ & 18 & 48 & matched sets & rating & $\nearrow$ & & & \\
\hline $\begin{array}{l}\text { Fieder et } \\
\text { al., } 2019^{c}\end{array}$ & 30 & 180 & continuous, (g)Imer & rating & $\varnothing$ & $\nearrow$ & $\begin{array}{c}\varnothing \text { (also for } \\
\text { coordinate errors) }\end{array}$ & $\swarrow$ \\
\hline $\begin{array}{l}\text { Morrison et } \\
\text { al., } 1992^{\mathrm{g}}\end{array}$ & 20 & 48 & $\begin{array}{l}\text { continuous, } \\
\text { multiple regression }\end{array}$ & rating & $\varnothing$ & & & \\
\hline $\begin{array}{l}\text { Woollams, } \\
2012^{\mathrm{h}}\end{array}$ & 16 & 80 & matched sets & rating & $\varnothing$ & $\varnothing$ & & \\
\hline $\begin{array}{l}\text { Rogers et } \\
\text { al., } 2015\end{array}$ & $\begin{array}{l}12 \text { older } \\
\text { adults }\end{array}$ & 48 & $\begin{array}{l}\text { matched sets, no } \\
\text { statistics reported }\end{array}$ & rating & & $\swarrow$ & & \\
\hline
\end{tabular}




\begin{tabular}{|c|c|c|c|c|c|c|c|c|}
\hline Study & $\begin{array}{l}\text { Participants } \\
(n)\end{array}$ & $\begin{array}{l}\text { Items } \\
(n)\end{array}$ & Design/analyses & Operationalisation & RT & Accuracy & Semantic errors & Omissions \\
\hline \multicolumn{9}{|c|}{ Distinctiveness } \\
\hline $\begin{array}{l}\text { Rabovsky } \\
\text { et al., } 2016\end{array}$ & 16 & 541 & continuous, (g)Imer & $\begin{array}{l}\text { feature-norm database (McRae et al., } \\
\text { 2005) }\end{array}$ & $\nearrow^{\mathrm{i}}$ & $\nearrow^{i}$ & & \\
\hline $\begin{array}{l}\text { Taylor et al., } \\
2012\end{array}$ & 31 & 345 & continuous, (g)Imer & $\begin{array}{l}\text { feature-norm database (McRae et al., } \\
\text { 2005) }\end{array}$ & $\nearrow^{j}$ & & & \\
\hline $\begin{array}{l}\text { Miozzo et } \\
\text { al., } 2015^{k}\end{array}$ & 17 & 146 & $\begin{array}{l}\text { continuous, } \\
\text { stepwise regression }\end{array}$ & $\begin{array}{l}\text { feature-norm database (McRae et al., } \\
\text { 2005) }\end{array}$ & & $\varnothing$ & & \\
\hline $\begin{array}{l}\text { Humphreys } \\
\text { et al., } 1988\end{array}$ & 20 & 76 & matched sets & $\begin{array}{l}\text { category distinctiveness ("structural } \\
\text { similarity"); rated features in common } \\
\text { with exemplars of a category (control } \\
\text { participants) }\end{array}$ & $\nearrow$ & & & \\
\hline
\end{tabular}

Note. RT = response time, (g)Imer = (generalised) linear mixed effects model analyses, RTs and accuracy: $\swarrow=$ poorer performance - slower RTs and

decreased accuracy with higher values of the measure, $\lambda=$ improved performance - faster RTs with increased accuracy and higher accuracy with higher

values of the variable, Semantic errors and omissions: $\swarrow=$ reduced numbers of errors of this type with higher values of the variable, $\nearrow=$ increased numbers

of errors of this type with higher values of the variable, $\varnothing=$ no effect, blank cells $=$ not investigated.

Participants - where not otherwise specified, young adults- typically undergraduate students.

a inhibitory effect on RT significant in a second round of naming.

${ }^{b}$ correlation measure based on 'intercorrelational strength' in Taylor et al. (2012).

c speeded naming paradigm (500ms deadline).

d results based on Holm-Bonferroni corrected $p$-values to account for testing for multiple variables of interest. 


\begin{tabular}{|c|c|c|c|c|c|c|c|c|}
\hline Study & $\begin{array}{l}\text { Participants } \\
(n)\end{array}$ & $\begin{array}{l}\text { Items } \\
(n)\end{array}$ & Design/analyses & Operationalisation & RT & Accuracy & Semantic errors & Omissions \\
\hline
\end{tabular}

e Bormann's (2011) measure was based on ratings capturing the number of category coordinates of a target, which was then dichotomised into words with

many and few competitors.

${ }^{\mathrm{f}}$ similar results in picture naming after familiarisation and a subsequent second round of naming.

${ }^{g}$ rated typicality in two categories: man-made versus naturally occurring objects.

h pre rTMS.

i distinctiveness added in additional analysis in discussion; non-significant when intercorrelational density was in the model at the same time.

${ }^{\mathrm{j}}$ naming was also faster for concepts with more highly correlated distinctive features.

${ }^{k}$ Miozzo et al.'s (2015) Specific Semantic Features measure contained two measures grouped through Principal Component Analysis: number of distinctive features and number of encyclopaedic features. 


\section{Number of semantic features}

Number of semantic features, often referred to as semantic richness, is a simple count of the total number of features generated by participants in response to a target word, for example when producing features for a feature-norm database (e.g., McRae et al., 2005). A higher number of features has been consistently found to facilitate both response times in picture naming and, where investigated, naming accuracy (Rabovsky et al., 2016, 2020; Taylor et al., 2012). Looking at specific types of semantic features (e.g., distinctive perceptual or shared functional features) Rico Duarte and Robert (2014) also reported a facilitatory effect from a higher number of semantic features.

\section{Intercorrelational Density}

Intercorrelational density captures the extent to which feature pairs of a concept co-occur across concepts. Rabovsky and colleagues $(2016,2020)$ reported lower naming accuracy for items with higher intercorrelational density. In contrast, there is inconsistent evidence regarding an effect on response latencies: While Rabovsky et al. (2016) found significantly slower latencies with increasing intercorrelational density, this effect only reached significance in a second repetition of the item set in Rabovsky et al., (2020), and was non-significant in Taylor et al. (2012).

\section{Number of near semantic neighbours}

Near semantic neighbours are defined as words that are semantically very similar to the target word and share many semantic features with it (e.g., a cosine similarity of at least 0.4 between feature vectors, Mirman, 2011). Increased numbers of near semantic neighbours resulted in less accurate picture naming accuracy and lead to more semantic errors and omissions (versus correct responses) under special conditions in a speeded picture naming task (a variant of simple picture naming designed to increase naming errors, Fieder et al., 2019; Mirman, 2011). Fieder et al. also found that a higher number of near semantic neighbours led to slower responses in speeded naming. However, Hameau et al. (2019), Lampe et al. (2017), and Bormann (2011, using a similar measure) found no significant effects in standard picture naming, on either errors or response latencies.

\section{Semantic similarity}


Fieder et al.'s (2019) semantic neighbourhood similarity measure captured the average similarity of a target word and its semantic neighbours, based on the cosine similarity of their feature vectors (note that in other research the term semantic similarity is used to refer to the relationship between a target and a stimulus context (e.g., Rose et al., 2019) or the semantic overlap between two concepts in a particular context (e.g., Rose \& Abdel Rahman, 2017)). Fieder et al. were the first to examine effects of semantic neighbourhood similarity on participants' picture naming performance but did so using speeded picture naming. They found that naming accuracy in a speeded picture naming task was reduced for words with higher semantic neighbourhood similarity (i.e., semantically more similar neighbours) and participants were more likely to make a semantic error, coordinate error, or an omission over a correct response. In contrast, the effect of semantic neighbourhood similarity on naming latencies was not significant.

\section{Typicality}

Typicality captures the extent to which a target word is representative of its taxonomic category. Previous studies investigating typicality in word production used ratings to determine concept typicality (e.g., Dell'Acqua et al., 2000; Holmes \& Ellis, 2006); however, it can also be obtained using feature norms (e.g., Rosch \& Mervis, 1975). Compared to the other semantic variables, a relatively large body of literature has investigated effects of rated typicality on picture naming latency and accuracy. Where significant effects of typicality on response times were found (Dell'Acqua et al., 2000; Grossman et al., 1998; Holmes \& Ellis, 2006; Jolicoeur et al., 1984), participants responded faster to words of higher typicality. However, note that significant effects on response latencies are not found consistently (see Fieder et al., 2019; Morrison et al., 1992; Woollams, 2012, for non-significant effects). For naming accuracy, Rogers et al. (2015) and Fieder et al. (speeded picture naming) reported conflicting results, while other studies reported non-significant results (Holmes \& Ellis, 2006; Woollams, 2012). However, the higher statistical power of Fieder et al.'s speeded naming study, the alignment of their effect with the facilitatory effect of typicality on response times in other studies, as well as the lack of statistical analyses by Rogers et al. make a strong case for a generally facilitatory effect of typicality. 


\section{Distinctiveness}

Distinctiveness indicates how special or unique the features of a concept are with regard to all other concepts of the database. Higher concept distinctiveness has been found to lead to faster (Rabovsky et al., 2016; Taylor et al., 2012) and more accurate responses (Rabovsky et al., 2016), although the effects in Rabovsky et al. were only significant once intercorrelational density was excluded from the analyses. Miozzo et al. (2015) investigated a similar measure, the number of distinctive features of a concept. The authors used Principal Component Analysis and found that this measure loaded on the same component, labelled Specific Semantic Features, as the number of encyclopaedic features. However, there was no evidence that this Specific Semantic Features component predicted naming latency in a stepwise regression analysis. Finally, also Humphreys et al. (1988) studied a related measure, structural similarity, which captures the extent to which a category is rated as having members which share their features. Participants were faster to name items from categories that were rated as having few shared features (the members of which were therefore presumably of higher average distinctiveness) in contrast to items from categories with many shared features (and therefore lower distinctiveness). While effects of distinctiveness were not assessed at the item level, the reported effect matches what Rabovsky et al. and Taylor et al. found for concept distinctiveness.

\section{Limitations of previous research}

This brief overview of the literature demonstrates that some semantic variables (e.g., typicality) have been investigated more than others (e.g., intercorrelational density). Despite this imbalance and the sparseness of the evidence base, previous research reporting significant effects of a single semantic variable has mostly provided a consensus on the direction of any observed effect (i.e., positive or negative effect), although some studies did not find conclusive (significant) effects. The occurrence of both significant and non-significant findings for some semantic variables could be due to experimental and methodological differences between the studies or shortcomings in their design. More specifically, studies differed widely in statistical power due to varying numbers of items and/or 
participants, characteristics of the participants (e.g., age), and experimental factors, such as the experimental language or the task design (e.g., speeded vs standard naming).

However, two crucial shortcomings apply to most previous studies: First, previous studies of effects of semantic variables on word production have focused on only one or two types of semantic variable at the same time. Importantly, this approach neglects the issue that word meaning is likely to be a multidimensional construct and that different aspects of word meaning probably each explain unique variance with their effects occurring simultaneously (Pexman et al., 2013). Hence, given that semantic variables would be expected to operate simultaneously and not selectively, they would also be expected to influence participants' picture naming behaviour simultaneously. Yet, despite this, previous research has not studied the joint effects of different types of semantic variables on the word production process.

Second, previous research has often insufficiently controlled for (non-semantic) psycholinguistic variables that are known to influence word production (e.g., Alario et al., 2004; Perret \& Bonin, 2019). This could have led to statistically significant effects of semantic variables in fact being artifacts of the lack of control. This is of particular concern given the intercorrelations within and between semantic and other psycholinguistic variables (e.g., words with more semantic features tend to have higher typicality and be of lower age of acquisition). Consequently, a significant effect of a semantic variable in the absence of sufficient control of psycholinguistic control variables and other semantic variables cannot confidently be interpreted as an independent effect of the variable of interest and the chance of a false positive finding, a Type 1 error, is increased. It is hence crucial that potentially confounding variables are controlled in the experimental design (e.g., via matching) or the statistical analyses (e.g., by using regression analyses) to avoid false positives. Only then can the unique effect of a variable of interest be identified with confidence. This was the aim of our research.

Two studies that deserve closer attention in the context of our research, are those of Rabovsky and colleagues (Rabovsky et al., 2016, 2020) who conducted behavioural and EEG analyses of number of semantic features and intercorrelational density. Rabovsky and colleagues $(2016,2020)$ improved 
on most previous research by studying these two semantic variables simultaneously. However, they only included familiarity, number of orthographic neighbours, and lexical frequency in their analyses (Rabovsky et al., 2016; visual complexity was additionally included in Rabovsky et al., 2020) and did not account for other psycholinguistic variables that influence word production (e.g., age of acquisition, name agreement, imageability, image agreement, Perret \& Bonin, 2019). However, the rigorous control of these variables is especially crucial when the effect of interest is potentially rather small.

Rabovsky et al. $(2016,2020)$ used McRae et al.'s (2005) feature database to derive stimuli and associated measures of number of semantic features and intercorrelational density. However, given that the studies were conducted with German-speaking participants, Rabovsky and colleagues translated the (English) database to German. While one can probably assume that cultural differences between English and German speakers would not have a dramatic impact on conceptual representations (and features) of the items in the database, some are undoubtedly problematic for a picture naming experiment in German. More specifically, some of the translated names for concepts have very low frequency in German and are unlikely to be elicited with high name agreement and other concepts are highly specific to North American culture (e.g., pie, gopher, cedar) (note however, that Rabovsky et al. (2016) familiarised half of their participants with the correct names before the experiment). Importantly, such differences may arise across languages, but may also influence performance of participants of different cultures with the same native language. Specifically, in this study we tested Australian English speakers, who may be as unfamiliar with some of the North American concepts as German participants. Thus, in the norming study presented in Study 1 we identified the items in the McRae et al.'s (2005) database that had high name agreement in Australian English, and selected these for Study 2, in order to reduce any impact that may have arisen due to cultural differences between speakers of American and Australian English.

Moreover, Rabovsky et al. $(2016,2020)$ used black and white photographs of the objects, which may have hindered recognition of some objects (e.g., lime vs lemon). Consequently, even though the studies by Rabovsky et al. $(2016,2020)$ provide some of the most comprehensive analyses 
to date, it remains unclear how reliable the effects are and, as in the other studies in the literature, whether some effects are miscalibrated (i.e., over- or underestimated) due to insufficient experimental control of psycholinguistic and semantic variables.

\section{This study}

In the light of the shortcomings of the previous studies to have explored semantic variables, we investigated the influences of an increased number (six) of different semantic variables on picture naming performance. This work aimed to determine if these semantic variables affect word production latency and accuracy by examining their effects simultaneously, while taking into account effects of relevant psycholinguistic variables. As outlined above, this knowledge will inform and advance word production theories by clarifying and dissociating effects of these semantic variables. Hence, this exploratory study both aims to make methodological contributions and to further extend and constrain theories of word production.

However, we also wished to ensure that our findings could be directly compared to the previous literature, rather than add another study with differing methodology. Consequently, as Rabovsky et al. (2016) provide one of the most rigorous investigations, we first replicate their analysis approach in order to directly compare our data to theirs and to test the reliability of their findings. Second, we incrementally increase the complexity of the analysis to ultimately, simultaneously, examine the effects of six feature-based semantic variables (number of near semantic neighbours, semantic similarity, number of semantic features, typicality, intercorrelational density, distinctiveness) while also controlling for important psycholinguistic variables known to affect picture naming. Hence, a, preregistered (Open Science Framework: https://osf.io/yw6ma/, Lampe et al., 2019), threefold approach to the analysis was used which conceptually replicates and extends Rabovsky et al.'s study. The three separate analyses addressed the following two research questions:

1. How is speech production affected by the number of semantic features ${ }^{1}$ and intercorrelational density of the semantic representation of the target word?

\footnotetext{
${ }^{1}$ This was termed featural richness in Rabovsky et al. (2016) and our preregistration.
} 
Analysis 1A: First, we conceptually replicated Rabovsky et al. (2016) and analysed effects of number of semantic features and intercorrelational density on naming latency and accuracy in English speaking participants, while controlling for concept familiarity, number of orthographic neighbours, and lexical frequency. In contrast to Rabovsky et al., we used only those McRae et al. (2005) items with high name agreement (in Australian English, as determined in Study 1). Moreover, we used an improved set of pictures (i.e., colour photographs instead of black and white pictures) and a slightly modified procedure (i.e., a familiarisation phase was used for half of the participants in the original study, while none of our participants were familiarised with any of the materials before the beginning of the task).

Analysis 1B: Subsequently, we sought to establish if any effects of number of semantic features and intercorrelational density in Analysis $1 \mathrm{~A}$ were statistical artifacts due to insufficient control of psycholinguistic variables. For this purpose, we extended Rabovsky et al.'s (2016) analysis (and Analysis 1A) by controlling for the psycholinguistic variables that were identified to influence picture naming by Perret and Bonin (2019): name agreement, image agreement, imageability, age of acquisition, conceptual familiarity, and lexical frequency. Moreover, we added the ordinal category position of an item (e.g., Howard et al., 2006) and the item's trial number in the experiment as independent variables (e.g., Baayen \& Milin, 2017).

2. How is speech production additionally affected by other semantic variables, specifically, the number of near semantic neighbours, semantic similarity, concept typicality, and distinctiveness, when the psycholinguistic variables in Analysis $1 \mathrm{~B}$ are also controlled for? If effects of the proposed semantic variables are significant, are they facilitative or inhibitory?

Analysis 2: In this analysis, we determined if, when studied simultaneously, any of the other feature-based semantic variables have effects on picture naming behaviour, and whether any effects of number of semantic neighbours and intercorrelational density are retained. We therefore analysed effects of the six semantic variables of interest simultaneously while controlling for the psycholinguistic variables detailed in Analysis 1B. 


\section{Study 1: Norming study}

Word and picture characteristics are known to influence performance in object naming studies (e.g., Alario et al., 2004; Perret \& Bonin, 2019). It is therefore important to account for such variables when studying effects of other variables of interest. Psycholinguistic control variables can be included in the analysis (e.g., as fixed effects), so that any variance in performance associated with them can be attributed to them and subtracted from the estimates of the variables of interest.

Some confounding variables are specific to the pictures or the population used in a study (e.g., name agreement) and can hence not be extracted from previously published norms. Therefore, in the absence of previous studies using these pictures, we collected normative data to be able to account for all psycholinguistic variables identified to influence picture naming behaviour in a Bayesian meta-analysis by Perret and Bonin (2019).

\section{Methods}

\section{Participants}

Name agreement, age of acquisition, and imageability ratings were collected from 45 Macquarie University undergraduate students ( 21 female; $M=20.2$ years old, range $=18-33$ years, $S D$ = 3.1) who were recruited from Macquarie University's Psychology participant pool and participated for course credit. A different population of 48 undergraduate students ( 42 female; $M=20.9$ years old, range $=17-35$ years, $S D=4.9)$ provided image agreement ratings. All participants were native Australian English speakers, right-handed and reported no history of neurological, cognitive, speech or language impairments. Participants were tested individually after giving written informed consent. The study was approved by Macquarie University's Human Ethics Committee.

\section{Stimuli, Material, and Procedure}

Colour photographs corresponding to all 541 items of the McRae et al. (2005) feature database were retrieved from the internet. If necessary, the pictures were edited, such that they showed the object on white background.

For the name agreement, age of acquisition, and imageability ratings, the pictures were divided into four lists containing 135 or 136 items each. Items from semantic categories were evenly 
distributed across the lists (e.g., buzzard, eagle, and vulture appeared in different lists). Items were presented in a randomised order, using Qualtrics software (Qualtrics, 2018).

Participants were asked to write the name of the pictures to obtain a measure of Australian name agreement (at least 11 participants per list). Subsequently, participants were presented with the items from one of the other lists as written words on the computer screen. In case of a word with ambiguous meaning (e.g., bat), a disambiguating description was provided together with the target word (e.g., bat (animal) vs bat (baseball)). Participants were asked to rate the age of acquisition and then the imageability of each item. The age of acquisition rating followed Johnston et al. (2010) and Gilhooly and Logie (1980): Participants were asked to estimate the age at which they thought they had first learned the name of the depicted object, choosing between seven age bands ranging from 0 to $13+$ years. For the imageability rating, participants had to estimate the item's capacity to arouse a mental image of an object on a 7-point Likert scale, following Cortese and Fugett (2004), Toglia and Battig (1978), and Paivio et al. (1968).

Image agreement ratings were obtained in an online study also using Qualtrics software. Each participant saw on average 307 of the 541 pictures in a randomised order (range $=104-330$ pictures, $S D=60.3)$. At least 22 participants rated each picture. The participants were asked to indicate the picture's image agreement following Snodgrass and Vanderwart (1980). Participants first saw a written word on the screen. Once ready, they advanced to a blank screen during which they were asked to create a mental image of the object they had read the name of. After 3 seconds, a single picture appeared on the screen and the participants were asked to judge how closely this picture matched their mental image of the object. Participants used a 5-point Likert scale, ranging from 1 "low agreement" to 5 "high agreement". Two additional options were given to indicate that the object was unknown or that the participant had thought of a different object when reading the word.

\section{Analysis and Results}

We will first detail how the item set for the picture naming study was selected based on the name agreement data. Subsequently, values of the other variables will be reported for that item subset with high name agreement. 
As we were interested in the name associated with an item rather than the spelling accuracy of production, responses with typing/spelling errors were corrected (e.g., achordian for target word accordion) and considered as correct responses. Items where less than $75 \%$ of participants produced the same response to a picture were then excluded from further analyses, which led to removal of 196 items. We retained four items where participants agreed on a different name to that used in the McRae et al. (2005) American English target list. For all of these items, we considered the appropriate Australian English labels for the same concept (i.e., "motorbike" for the target word "motorcycle", "pram" for the target word "buggy", "prawn" for the target word "shrimp", and "teacup" for "cup").

From the remaining 345 items we discarded items when the agreed name:

1. was another target word (e.g., $82 \%$ of participants used the target word "church" to name the picture of a chapel).

2. was a superordinate (e.g., $91 \%$ of participants used "bird" to name the picture of a partridge).

3. referred to a different concept that was not another target (e.g., $91 \%$ used "wolf" to name the picture of a coyote).

This process led to a final set of 297 items. For each of these items, average age of acquisition, imageability, and image agreement ratings were calculated. For the image agreement ratings, responses where a participant had indicated that the word was unknown or that they had thought of a different object were excluded from the analysis. The characteristics of the 297 items are listed in Table 2. 


\section{Table 2}

Descriptive statistics for name agreement (proportion of participants providing the target name) and rated variables (age of acquisition, imageability, and image agreement) for the final 297 items set

\begin{tabular}{lcccc}
\hline & $\begin{array}{c}\text { Name agreement (pro- } \\
\text { portion of participants) }\end{array}$ & $\begin{array}{c}\text { Age of acquisition } \\
\text { (7 age bands) }\end{array}$ & $\begin{array}{c}\text { Imageability } \\
\text { (7-point scale) }\end{array}$ & $\begin{array}{c}\text { Image } \\
\text { agreement } \\
\text { (5-point scale) }\end{array}$ \\
\hline Average & 0.94 & 3.21 & 5.43 & 4.36 \\
Minimum & 0.75 & 1.25 & 2.09 & 2.91 \\
Maximum & 1.00 & 6.64 & 6.64 & 5.00 \\
SD & 0.07 & 1.03 & 0.73 & 0.42 \\
\hline
\end{tabular}

\section{Study 2: Picture naming experiment}

A standard continuous picture naming experiment using simple picture naming without context manipulation was conducted to examine the effect of semantic variables on word production processes. The experimental procedure of this study and the three main analyses were preregistered on the Open Science Framework (Lampe et al., 2019; https://osf.io/yw6ma/) and the data and analyses scripts are available there. The materials are available on request from the corresponding author. The study was approved by the Macquarie University Human Ethics Committee.

\section{Methods}

\section{Participants}

Participants were recruited from Macquarie University's Psychology participant pool and received course credit or monetary compensation (AUD15 per hour) for their time. All participants provided informed consent. Participants were eligible to participate in this study if they were Australian English native speakers, were right-handed, 17-35 years old, and had normal or correctedto-normal vision. Exclusion criteria were a history of neurological or cognitive impairments or a history of speech and language impairments. 89 participants took part in this study, of whom 2 were excluded because they did not fulfil the eligibility criteria or did not comply with the instructions. The data from 87 participants ( 68 female; $M=20.1$ years old, range $=17-33$ years, $S D=2.3$ ) were therefore used in the analyses.

\section{Stimuli}


The stimuli consisted of pictures of the 297 items from the McRae et al. (2005) database that were retained following the norming process described in Study 1 above. Another feature database (Devereux et al., 2014) provided information on semantic categories. We used the categories assigned by Devereux et al. for the 267 of our items that appeared in this database and assigned the remaining items to one of Devereux et al.'s 24 semantic categories. As 45 of our items were allocated to the "miscellaneous" category by Devereux et al., we re-assigned these items to new categories where possible (e.g., 10 items (e.g., skis) to a category of "sport equipment", and 7 items (e.g., pipe) to a category of "plumbing"). This procedure led to 35 different semantic categories, containing between 1 and 32 items ( $M=8.49$ items per category).

Stimuli were divided into four blocks (Blocks 1-4). Block 1 was designed as a set of stimuli that would not be prone to effects of cumulative semantic inhibition (i.e., the finding that the production of a target word is slower the more items from the same semantic category were previously named in the experiment; e.g., Howard et al., 2006) by comprising items from different semantic categories. This block consisted of 35 items, nine of which came from the miscellaneous category and one item of each of the different semantic categories, under the condition that no items in this block were near semantic neighbours (feature vector similarity cosine between pairs of items <.4; Mirman, 2011). The remaining items were divided evenly between Blocks $2-4$ (Blocks 2 and $3 n=87$, Block $4 n=88$ items). Three pseudorandomised orders of items within each of the four blocks were created. For Blocks 2-4 a minimum of two items from different semantic categories intervened between items of the same semantic category in order to reduce interference/facilitation from closely related items. Finally, six different experimental lists were created by manipulating the order and pseudorandomisations of the different blocks. Block 1, controlling for the cumulative semantic inhibition effect, always appeared in the first position in each list, while Blocks 2-4 were presented in varying orders afterwards (i.e., positions 2-4, see Appendix A). Each participant saw one of the pseudorandomised experimental lists.

For all 297 items, information was available for six psycholinguistic control variables as suggested by Perret and Bonin (2019) to influence picture naming: name agreement, image 
agreement, imageability, age of acquisition, familiarity (all values obtained from our norming study), and frequency (Zipf, based on television subtitles, SUBTLEX-UK; van Heuven et al., 2014). In addition, to replicate Rabovsky et al.'s (2016) analysis, the number of orthographic neighbours of the items was retrieved from the N-Watch database (Coltheart's N; Davis, 2005). Two further control variables were based on the experimental lists: A measure accounting for the number of previously seen items of the same semantic category to control for the cumulative semantic inhibition effect (Howard et al., 2006) and the rank-order of an item within the experiment to account for habituation to the experimental situation or fatigue (Baayen \& Milin, 2010).

Information on six feature-based semantic predictor variables was derived from information given in McRae et al. (2005): number of semantic features, intercorrelational density, number of near semantic neighbours, semantic similarity, typicality, and distinctiveness.

Number of semantic features was a simple count of the semantic features generated for a target word (e.g., Rabovsky et al., 2016). For intercorrelational density, the shared variance of a concept's correlated feature pairs was determined and then summed (e.g., Rabovsky et al., 2016). To qualify as a near semantic neighbour, the cosine feature vector similarity with the target had to be at least .4 (Hameau et al., 2019; Mirman, 2011; Mirman \& Graziano, 2013). Following Mirman and Magnuson (2008), we operationalised semantic similarity as the average similarity between the feature vectors of the target and all other words in McRae et al.'s (2005) feature database (note that the only previous investigation of semantic similarity in word production (Fieder et al., 2019) excluded from the calculation concepts that had a feature vector similarity of 0 with the target. Other studies that have used measures of semantic similarity, are not relevant here as they have investigated different issues, e.g., the relationship between a target word and the stimulus context (e.g., Rose et al., 2019) or the semantic overlap between two concepts in a context (e.g., Rose \& Abdel Rahman, 2017)). Typicality was calculated in a similar way to Rosch and Mervis' (1975) family resemblance score: Each feature of an item received a score based on the number of other items in the same semantic category that were credited with that particular feature. This feature weight was then divided by the number of items in the semantic category and ultimately multiplied by its production frequency (number of participants 
who produced that feature for the item) before the feature weights of all features of an item were summed. Finally, distinctiveness was the inverse of the number of concepts in which a certain feature occurs across the database, which was then averaged across the features of a concept (e.g., Rabovsky et al., 2016). See Appendix B for a more detailed description of the calculation of the semantic variables and Appendix $C$ for a demonstration that the variability of the semantic variables in the item set selected for this study was comparable to the full feature database by McRae et al. (2005).

\section{Procedure}

Testing took place in a quiet room at Macquarie University. In addition to naming latency and accuracy, a continuous EEG signal was recorded using a 64 channels ActiveTwo BioSemi system (BioSemi, Amsterdam, The Netherlands). The EEG data are reported elsewhere (Lampe, Bürki, et al., 2021). The simple picture naming task analysed here was the first task in a larger study that involved the participants subsequently naming the same pictures twice more in different experimental paradigms (e.g., Lampe, Hameau, et al., 2021).

Picture presentation and trial-sequence were controlled by Presentation ${ }^{\circledR}$ software (Version 20.0, Neurobehavioral Systems, Inc., Berkeley, CA, www.neurobs.com). Trial sequence was as follows: First a fixation cross appeared in the centre of the screen for a random duration of between 500 and $1000 \mathrm{~ms}$, such that participants would be unable to predict the exact onset of the picture. Next, a picture was displayed for $2000 \mathrm{~ms}$ on a white background in the centre of the screen and the participants were instructed to name the picture as quickly and accurately as possible, using a single word only. After the picture offset the screen was blank for $1000 \mathrm{~ms}$ before the start of the next trial.

The experiment was presented on a Dell Precision tower 3620 running Windows 10 and using an AOC FreeSync LED monitor. Verbal responses and response latencies were recorded using a voice trigger in Presentation ${ }^{\circledR}$ and a Behringer preamplifier (Tube Ultragain Mic100) together with a Rode NTG1 shotgun microphone. The keyboard was used to navigate through the experiment.

The experiment began with 5 practice trials in which participants named pictures that were not part of the 297 experimental stimuli and came from different semantic categories to the experimental stimuli. There was a break after the practice phase and after each experimental block for 
the participants to ask questions and to rest. The first trial after each break included another practice picture, which again came from a different semantic category from all experimental stimuli. The task took about 30 minutes.

\section{Response coding}

After the experiment, all responses were transcribed and coded for naming accuracy. Response latencies recorded by the voice trigger were checked and manually adjusted as necessary by visual and auditory inspection of the waveform using the program Praat (Version 6.0.49; Boersma \& Weenink, 2019).

Accuracy of the first response was coded. Responses were coded as correct if the first response consisted of (only) the correctly pronounced target word. Only correct responses were considered for the naming latency analyses. For the accuracy analyses, in addition to correct responses, responses with a determiner preceding the correct name (e.g., "an octopus"), disfluencies on the target's initial phoneme (e.g., "sss sofa"), hesitations (e.g.,"erm apple"), and elaborations (e.g., "yellow truck" for "truck") were coded as correct. Responses consisting of a synonym or acceptable response (e.g., "sofa" for "couch"), abbreviations (e.g., "croc" for "crocodile"), or a response continuing from the previous item with subsequent correct naming of the target word (e.g., "emu .. bed" for "bed" with "emu" being the previous item) were coded as 'not analysed' (NA) and excluded from the analyses. Disfluencies with self-corrections (e.g., "oct squid" for "squid"), incomplete responses (e.g., "so" for "sofa"), incorrect responses (e.g., "chair" for "sofa"), as well as instances where the participants did not respond or made a comment that expressed a failure to respond (e.g., "I don't know"; i.e., omissions) were coded as errors (see Appendix D, for examples and a summary of the coding system).

\section{Data analysis}

The data was analysed in RStudio (Version 1.2.5033; RStudio Team, 2019) using (generalised) linear mixed effect models ((G)LMMs) as implemented in the Ime4-package (Version 1.1.21; Bates et al., 2017). $p$-values were derived using ImerTest (Version 3.1.1; Kuznetsova et al., 2017). For the specification of the random effects structure we followed Bates et al. (2015) and the model fit of 
nested models was assessed using likelihood ratio tests (stats package, Version 3.6.1; R Core Team, 2018). Plots were created using the package sjPlot (Version 2.6.3; Lüdecke, 2019).

Ten data points from two participants had to be removed because of a programming error. Moreover, four items (board, bridge, racquet, and pie) were excluded from the analyses as many participants produced an elaboration that comprised a subordinate of the target word (e.g., tennis racquet). Outliers were identified for naming latency and accuracy, both for participants and items based on visual inspection of boxplots. Two participants who performed considerably less accurately than the other participants ( $69 \%$ and $62 \%$ naming accuracy versus mean accuracy of $86 \%, S D=5 \%$ ) were excluded from further analyses. Two items (crowbar and raft) were identified as outliers for naming accuracy (26\% and $34 \%$ accuracy versus mean accuracy of $86 \%$; $S D=15 \%$ ) and excluded from further analyses.

For the naming latency analyses, only correct responses were considered and all other responses (3,782 data points, $15.30 \%$ of the data) were disregarded. 20,943 data points from 291 items and 85 participants entered the analyses, and mean naming latency was $900.81 \mathrm{~ms}$. For naming accuracy, responses coded as NA (synonyms, acceptable alternatives, abbreviations, and continuing responses; see section Response coding) were not analysed (171 data points, $0.69 \%$ of the data). 24,554 data points from 291 items and 85 participants entered the accuracy analyses, of which $85.69 \%$ were correct responses.

All predictor variables were standardised using a z-transformation ${ }^{2}$. Based on the output of the boxcox function (EnvStats Version 2.3.1; Millard, 2013), naming latency was negative reciprocally transformed to approximate a normal distribution (note that the negative reciprocal transformation preserves order among values of the same sign).

Three separate analyses on response latencies and naming accuracy were conducted:

Analysis 1A: Replication of Rabovsky et al. (2016). In this analysis, the number of semantic features and intercorrelational density were the only semantic variables included in the model.

\footnotetext{
${ }^{2}$ The standardisation of ordinal category position and item number in the experiment was not preregistered. However, standardisation of all variables was necessary to facilitate model convergence.
} 
Following Rabovsky et al. (2016), we also included rated familiarity, number of orthographic neighbours, and lexical frequency. We also replicated Rabovsky et al.'s random effects structure by initially including random intercepts for participants and items as well as random slopes for participants for both semantic variables. However, random slopes were only retained in the models if they were supported by the data, following the model definition approach described by Bates et al. (2015).

\section{Analysis 1B: Replication of Rabovsky et al. (2016) including more psycholinguistic} control variables. In this analysis, the number of semantic features and intercorrelational density remained the only semantic predictor variables included in the model. However, in contrast to Rabovsky et al. (2016), we included a wider range of psycholinguistic control variables in the analysis. Following Perret and Bonin (2019) and Baayen and Milin (2017), the models included the control variables name agreement, image agreement, imageability, age of acquisition, familiarity, frequency, as well as a measure of ordinal category position and rank-order of an item within the list. Number of orthographic neighbours from Analysis 1A was not included. Again, random intercepts for participants and items were included in the models as well as random slopes for the two semantic variables by participants, which were retained if they were supported by the data.

\section{Analysis 2: Extension of Rabovsky et al.'s (2016) analyses to include six semantic}

variables. In the final analyses, the models included four further semantic variables (number of near semantic neighbours, semantic similarity, typicality, and distinctiveness), in addition to number of semantic features and intercorrelational density and the psycholinguistic control variables described in Analysis 1B. Random intercepts for participants and items were included in the models as well as random slopes for semantic variables by participants if they were supported by the data.

\section{Results}

\section{Correlations}

Pearson's correlations between variables were determined (stats package Version 3.6.1; R Core Team, 2018) to investigate potential multicollinearity. Hutcheson and Sofroniou (1999) suggested that correlation coefficients of larger than .80 indicate multicollinearity. While most correlations between 
semantic predictor variables were significant (Table 3), the observed coefficients were not indicative of any problematic levels of collinearity between variables. In addition, we calculated variance inflation factors (VIF) for the fixed effects of each (G)LMM in the analyses to further identify any possibly problematic levels of multicollinearity between the variables entered in the individual analyses. The focus of those calculations were the semantic predictor variables, and not the control variables. VIFs for the semantic predictors in all models (Tables 4-6) were below the values that have been recommended as acceptable levels (depending on the author, VIFs $>2.5$ (Allison, 2012), or around 5 (Hair et al., 2014; Rogerson, 2011) indicate potentially problematic multicollinearity). Hence, multicollinearity was not a problem between the predictors of the models used in the analyses. 


\section{Table 3}

Pearson's correlations between the semantic predictor variables and the psycholinguistic control variables

\begin{tabular}{|c|c|c|c|c|c|c|}
\hline Variable & $\begin{array}{c}\text { Number near } \\
\text { semantic } \\
\text { neighbours }\end{array}$ & $\begin{array}{l}\text { Semantic } \\
\text { similarity }\end{array}$ & $\begin{array}{l}\text { Number } \\
\text { semantic } \\
\text { features }\end{array}$ & Typicality & $\begin{array}{l}\text { Intercorrelational } \\
\text { density }\end{array}$ & Distinctiveness \\
\hline Semantic similarity & $0.58^{\star \star \star}$ & & & & & \\
\hline Number semantic features & -0.08 & -0.01 & & & & \\
\hline Typicality & $0.37^{* * *}$ & $0.14^{*}$ & $0.25^{* * *}$ & & & \\
\hline Intercorrelational density & $0.42^{\star \star *}$ & 0.07 & $0.40^{* \star *}$ & $0.44^{\star \star \star}$ & & \\
\hline Distinctiveness & $-0.51^{\star \star *}$ & $-0.58^{\star \star \star}$ & 0.03 & $-0.25^{\star * *}$ & $-0.41^{\star * \star}$ & \\
\hline Name agreement & -0.07 & -0.04 & -0.01 & -0.05 & -0.07 & 0.04 \\
\hline Age of acquisition & 0.05 & 0.04 & $-0.20^{\star \star \star}$ & $-0.14^{*}$ & $-0.19^{* \star \star}$ & 0.09 \\
\hline Imageability & -0.05 & -0.08 & $0.24^{\star \star *}$ & $0.12^{*}$ & $0.16^{\star \star}$ & -0.02 \\
\hline Image agreement & $0.31^{* \star *}$ & $0.24^{\star \star \star}$ & 0.03 & 0.04 & $0.12^{\star}$ & $-0.19^{\star \star \star}$ \\
\hline Frequency & $-0.22^{\star \star \star}$ & -0.11 & $0.24^{* * *}$ & 0.03 & 0.09 & 0.09 \\
\hline Familiarity & $-0.27^{\star \star \star}$ & $-0.37^{\star \star *}$ & $0.15^{\star}$ & $0.12^{*}$ & 0.05 & $0.20^{* \star *}$ \\
\hline Number orthographic neighbours & $-0.12^{\star}$ & 0.03 & 0.03 & -0.04 & -0.04 & 0.05 \\
\hline
\end{tabular}




\section{Analysis 1A: Replication of Rabovsky et al. (2016)}

Naming latency. This analysis replicated the analysis run by Rabovsky et al. (2016) and, therefore, we fitted a model including the same control and semantic variables as in that paper. Following Bates et al.'s (2015) approach to define a random effects structure that is supported by the data, we identified a model with number of semantic features as random slope for participants, including correlations between the random slope and the intercept, as the model explaining the data best (Table 4, Model 1A.1). Responses were faster for words that were more familiar and had higher word frequency, however, the two semantic variables number of semantic features and intercorrelational density did not reach significance (see also Figure E1, Panel A, for a graphical display of the fixed effects and their confidence intervals).

Naming accuracy. To replicate the analysis of Rabovsky et al. (2016), we used a model with the same fixed effects structure as for the naming latency analysis of Analysis 1A.1. A model with crossed random intercepts for items and participants and no random slope provided the best fit of the data (Table 4, Model 1A.2). Increasing familiarity and frequency were found to facilitate naming accuracy. Moreover, response accuracy was facilitated by a higher number of semantic features (see also Figure E1, Panel B). 


\section{Table 4}

Analysis 1 A replicating Rabovsky et al. (2016): Summarised output of linear mixed model analysis of picture naming latency (Models 1A.1) and naming accuracy (Model 1A.2)

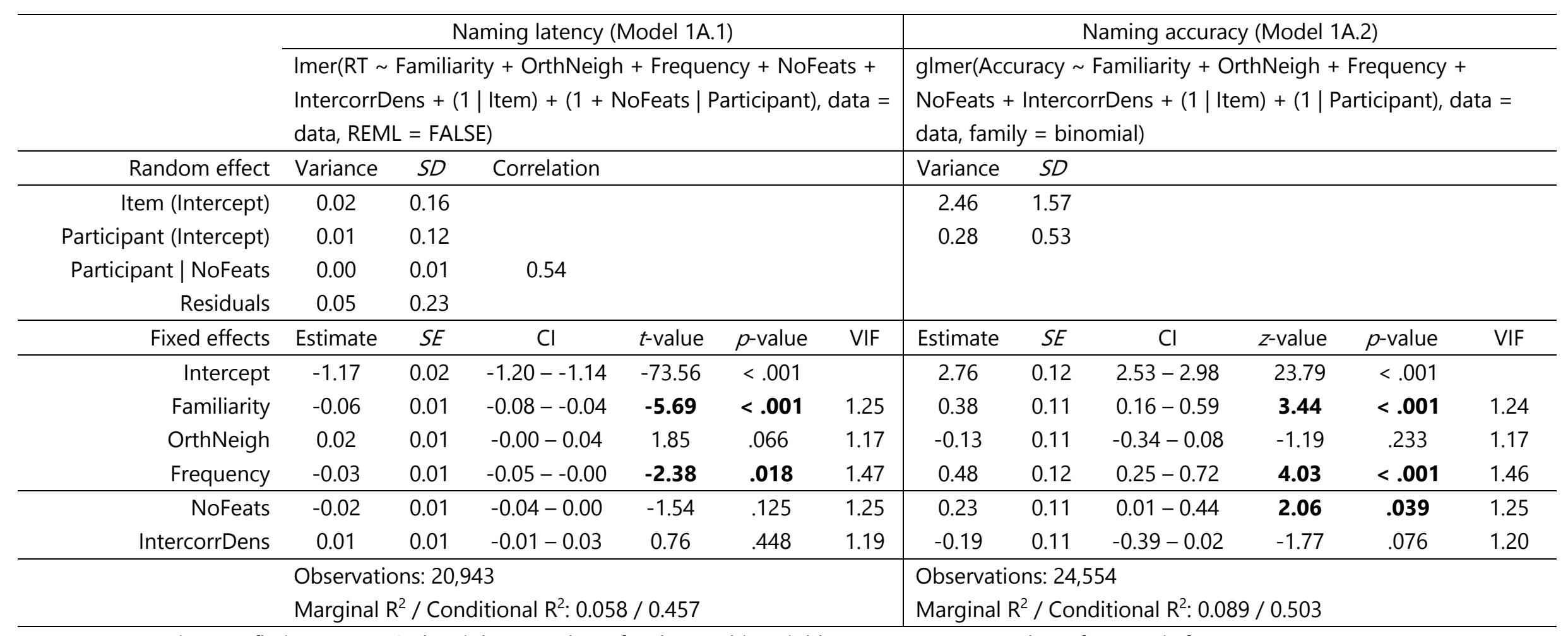

Note. VIF = Variance Inflation Factor, OrthNeigh = Number of orthographic neighbours, NoFeats = number of semantic features, IntercorrDens =

intercorrelational density, Participant $\mid X=$ random slope of $X$ by participants;

values for significant effects $(p<.05)$ are printed in bold. 


\section{Analysis 1B: Replication of Rabovsky et al. (2016) including more psycholinguistic control}

\section{variables}

Naming latency. We extended the original Rabovsky et al. (2016) analyses by including more control variables, while the semantic variables remained the same. Again, a model with a byparticipants random slope for number of semantic features, including the correlation between the random slope and intercept, provided the best fit of the data (Table 5, Model 1B.1).

Most control variables had a significant effect on response latencies and their effects pointed in the expected directions: Responses were faster for words with higher name agreement, higher image agreement, and higher familiarity, and responses were slower for words acquired later in life and the more items from the same category that had been previously seen in the experiment (Cumulative Semantic Interference effect). Finally, responses became slower over the course of the experiment. Neither of the two semantic variables showed significant effects on naming latency (Table 5, Figure E2, Panel A).

Naming accuracy. In replicating Rabovsky et al.'s (2016) experiment, we extended Analysis 1A.2 with further control variables (as in Analysis 1B.1). The full complex effect structure was not supported by the data, and a model without by-participant random slopes provided the best fit (Table 5, Model 1B.2).

All control variables reached significance, except for familiarity, and their effects were in the expected directions: Higher name agreement, image agreement, imageability, and frequency facilitated naming accuracy, while higher age of acquisition and a higher number of items from the same semantic category previously seen in the experiment (Cumulative Semantic Interference effect) led to less accurate responses. Moreover, participants were more accurate as the experiment progressed. The two semantic variables were also significant: While higher numbers of features improved naming accuracy, higher intercorrelational density reduced naming accuracy (Table 5, Figure E2, Panel B). 


\section{Table 5}

Analysis 1 B replicating Rabovsky et al. (2016) taking more psycholinguistic control variables into account: Summarised output of linear mixed model analysis of picture naming latency (Models 1B.1) and naming accuracy (Model 1B.2)

\begin{tabular}{|c|c|c|c|c|c|c|c|c|c|c|c|c|}
\hline & \multicolumn{6}{|c|}{ Naming latency (Model 1B.1) } & \multicolumn{6}{|c|}{ Naming accuracy (Model 1B.2) } \\
\hline & \multicolumn{6}{|c|}{$\begin{array}{l}\text { Imer(RT NameAgr + ImageAgr + Imageability + AoA + } \\
\text { Familiarity + Frequency + OrdCatPos + Order + NoFeats + } \\
\text { IntercorrDens + (1| Item })+(1+\text { NoFeats | Participant }) \text {, data, } \\
\text { REML = FALSE })\end{array}$} & \multicolumn{6}{|c|}{$\begin{array}{l}\text { glmer(Accuracy NameAgr + ImageAgr + Imageability + AoA } \\
+ \text { Familiarity + Frequency + OrdCatPos + Order + NoFeats + } \\
\text { IntercorrDens + (1| Item })+(1 \mid \text { Participant }) \text {, data, family }= \\
\text { binomial) }\end{array}$} \\
\hline Random effect & Variance & $S D$ & Correlation & & & & Variance & $S D$ & & & & \\
\hline Item (Intercept) & 0.02 & 0.13 & & & & & 1.43 & 1.20 & & & & \\
\hline Participant (Intercept) & 0.01 & 0.12 & & & & & 0.28 & 0.53 & & & & \\
\hline Participant | NoFeats & 0.00 & 0.01 & 0.53 & & & & & & & & & \\
\hline Residuals & 0.05 & 0.23 & & & & & & & & & & \\
\hline Fixed effects & Estimate & $S E$ & $\mathrm{Cl}$ & $t$-value & $p$-value & VIF & Estimate & $S E$ & $\mathrm{Cl}$ & $\begin{array}{c}z- \\
\text { value }\end{array}$ & $p$-value & VIF \\
\hline Intercept & -1.17 & 0.01 & $-1.20--1.14$ & -78.68 & $<.001$ & & 2.73 & 0.10 & $2.53-2.92$ & 27.50 & $<.001$ & \\
\hline NameAgr & -0.06 & 0.01 & $-0.07--0.04$ & -6.99 & $<.001$ & 1.11 & 0.72 & 0.08 & $0.57-0.88$ & 9.11 & $<.001$ & 1.08 \\
\hline ImageAgr & -0.05 & 0.01 & $-0.07--0.04$ & -6.44 & $<.001$ & 1.17 & 0.34 & 0.08 & $0.18-0.51$ & 4.04 & $<.001$ & 1.20 \\
\hline Imageability & -0.02 & 0.01 & $-0.04-0.00$ & -1.91 & .058 & 1.82 & 0.26 & 0.11 & $0.05-0.47$ & 2.46 & .014 & 1.78 \\
\hline AoA & 0.03 & 0.01 & $0.01-0.05$ & 2.53 & .012 & 2.30 & -0.31 & 0.12 & $-0.54--0.07$ & -2.59 & .010 & 2.27 \\
\hline Familiarity & -0.04 & 0.01 & $-0.06--0.02$ & -4.44 & $<.001$ & 1.44 & 0.14 & 0.09 & $-0.04-0.32$ & 1.54 & .122 & 1.43 \\
\hline Frequency & -0.02 & 0.01 & $-0.03-0.00$ & -1.59 & .114 & 1.54 & 0.27 & 0.10 & $0.08-0.46$ & 2.80 & .005 & 1.53 \\
\hline OrdCatPos & 0.01 & 0.00 & $0.00-0.02$ & 3.17 & .002 & 3.11 & -0.13 & 0.05 & $-0.24--0.03$ & -2.59 & .010 & 3.06 \\
\hline Order & 0.01 & 0.00 & $0.00-0.01$ & 2.20 & .028 & 3.08 & 0.11 & 0.05 & $0.02-0.20$ & 2.52 & .012 & 3.01 \\
\hline NoFeats & -0.02 & 0.01 & $-0.03-0.00$ & -1.70 & .089 & 1.28 & 0.21 & 0.09 & $0.04-0.38$ & 2.38 & .017 & 1.29 \\
\hline IntercorrDens & 0.01 & 0.01 & $-0.00-0.03$ & 1.45 & .147 & 1.24 & -0.22 & 0.09 & $-0.39--0.06$ & -2.61 & .009 & 1.27 \\
\hline
\end{tabular}


Observations: 20,943

Marginal $R^{2}$ / Conditional $R^{2}: 0.139$ / 0.454

Observations: 24,554

Marginal $R^{2}$ / Conditional $R^{2}: 0.231$ / 0.494

Note. VIF = Variance Inflation Factor, NameAgr = name agreement, ImageAgr = image agreement, AoA = age of acquisition, OrdCatPos = ordinal category

position, NoFeats = number of semantic features, IntercorrDens = intercorrelational density, Participant $\mid X=$ random slope of $X$ by participants;

values for significant effects $(p<.05)$ are printed in bold. 


\section{Analysis 2: Extension of Rabovsky et al. 's (2016) analyses to include six semantic variables}

Naming latency. In this analysis, we added the four additional semantic variables number of near semantic neighbours, semantic similarity, typicality, and distinctiveness to Analysis 1B.1. A model with random by-participant slopes for semantic similarity, number of near semantic neighbours, and number of semantic features, including correlations between the slopes and with the intercept provided the best fit of the data (Table 6, Model 2.1).

Effects of the control variables (see Table 6) were comparable to the effects found in Analysis 1B.1 with faster responses for words with higher name agreement, higher image agreement, and higher familiarity. Responses were slower for words with a higher age of acquisition, the more items from the same category were previously seen in the experiment (Cumulative Semantic Interference effect), and responses slowed over the course of the experiment. Importantly, the number of semantic features, as well as the distinctiveness of an item, significantly predicted naming latency: Responses were faster for items with more features and slower the higher the distinctiveness of an item.

These findings are displayed in Figure 1 (Panel A), where red lines (left of centre) indicate a facilitatory effect of a variable with faster responses as that variable increases in value, while blue lines (right of centre) indicate an inhibitory effect with slower responses as that variable increases in value. Confidence intervals that cross the black zero line are indicative of non-significant effects.

Naming accuracy. When the four additional semantic variables number of near semantic neighbours, semantic similarity, typicality, and distinctiveness were added to Analysis 1B.2, a model with a by-participant random slope for number of near semantic neighbours and its correlation with the intercept provided the best fit of the data (Table 6, Model 2.2).

Effects for the control variables (Table 6) remained comparable to Analysis 1B.2, with higher name agreement, image agreement, imageability, and frequency facilitating naming accuracy, and higher age of acquisition and a higher number of items from the same semantic category previously seen in the experiment (Cumulative Semantic Interference effect) leading to more naming errors. Moreover, participants' accuracy increased over the course of the experiment. Only two semantic 
variables reached significance: Higher numbers of semantic features led to more correct responses, while higher intercorrelational density led to reduced naming accuracy.

The findings are plotted in Figure 1 (Panel B), where red lines (right of centre) indicate a facilitatory effect with more accurate responses with higher values of a variable, while blue lines (left of centre) indicate an inhibitory effect with reduced naming accuracy with higher values of a variable. Again, confidence intervals that cross the black zero line are indicative of non-significant effects.

\section{Figure 1}

Analysis 2 extending Rabovsky et al. (2016) with other semantic variables: Fixed effects estimates with 95\% confidence interval of picture naming latency analysis (Panel A; Model 2.1) and accuracy analysis (Panel B; Model 2.2)

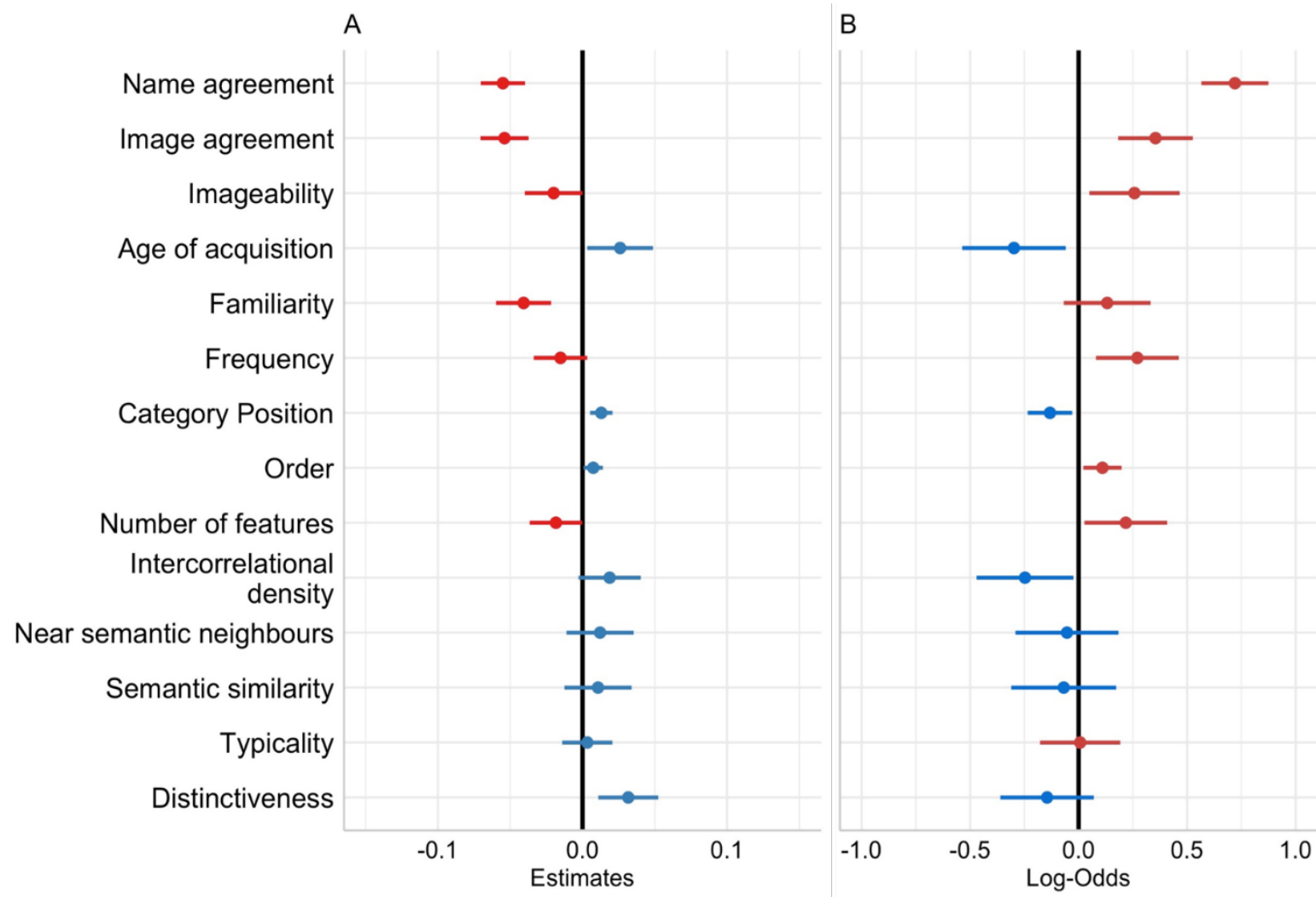

Note. Panel A shows the output of the naming latency analysis and Panel B the output of the naming accuracy analysis; red lines (to the left of centre for latency, and right for accuracy) indicate increased values of the variable lead to better performance, and blue lines (to the right of centre for latency and left for accuracy) indicate worse performance. 


\section{Table 6}

Analysis 2 extending Rabovsky et al. (2016) with other semantic variables: Summarised output of linear mixed model analysis of picture naming latency (Model 2.1) and naming accuracy (Model 2.2)

\begin{tabular}{|c|c|c|c|c|c|c|c|c|c|c|c|c|}
\hline & \multicolumn{6}{|c|}{ Naming latency (Model 2.1) } & \multicolumn{6}{|c|}{ Naming accuracy (Model 2.2) } \\
\hline & \multicolumn{6}{|c|}{$\begin{array}{l}\text { Imer(RT NameAgr + ImageAgr + Imageability + AoA + } \\
\text { Familiarity + Frequency + OrdCatPos + Order + NoFeats + } \\
\text { IntercorrDens + NrSemNeigh + SemSim + Typicality + } \\
\text { Distinct + (1 | Item })+(1+\text { NoFeats + NrSemNeigh + } \\
\text { SemSim | Participant }), \text { data = data, REML = FALSE })\end{array}$} & \multicolumn{6}{|c|}{$\begin{array}{l}\text { glmer(Accuracy NameAgr + ImageAgr + Imageability + } \\
\text { AoA + Familiarity + Frequency + OrdCatPos + Order + } \\
\text { NoFeats + IntercorrDens + NrSemNeigh + SemSim + } \\
\text { Typicality + Distinct + }(1 \mid \text { Item })+(1+\text { NrSemNeigh } \mid \\
\text { Participant }), \text { data }=\text { data, family = binomial })\end{array}$} \\
\hline Random effect & Variance & $S D$ & Correlation & & & & Variance & $S D$ & Correlation & & & \\
\hline Item (Intercept) & 0.02 & 0.12 & & & & & 1.42 & 1.19 & & & & \\
\hline Participant (Intercept) & 0.01 & 0.12 & & & & & 0.28 & 0.53 & & & & \\
\hline Participant | NoFeats & 0.00 & 0.01 & 0.50 & & & & & & & & & \\
\hline $\begin{array}{r}\text { Participant | } \\
\text { NrSemNeigh }\end{array}$ & 0.00 & 0.02 & -0.24 & 0.02 & & & 0.02 & 0.15 & 0.34 & & & \\
\hline Participant | SemSim & 0.00 & 0.01 & 0.07 & -0.61 & -0.49 & & & & & & & \\
\hline Residuals & 0.05 & 0.23 & & & & & & & & & & \\
\hline Fixed effects & Estimate & SE & $\mathrm{Cl}$ & $t$-value & $p$-value & VIF & Estimate & SE & $\mathrm{Cl}$ & $\begin{array}{c}z_{-}^{-} \\
\text {value }\end{array}$ & $p$-value & VIF \\
\hline (Intercept) & -1.17 & 0.02 & $-1.20--1.14$ & -78.43 & $<.001$ & & 2.73 & 0.10 & $2.54-2.92$ & 27.67 & $<.001$ & \\
\hline NameAgr & -0.06 & 0.01 & $-0.07--0.04$ & -7.07 & $<.001$ & 1.11 & 0.72 & 0.08 & $0.57-0.87$ & 9.13 & $<.001$ & 1.08 \\
\hline ImageAgr & -0.05 & 0.01 & $-0.07--0.04$ & -6.37 & $<.001$ & 1.27 & 0.35 & 0.09 & $0.18-0.53$ & 4.03 & $<.001$ & 1.30 \\
\hline Imageability & -0.02 & 0.01 & $-0.04--0.00$ & -1.96 & .051 & 1.86 & 0.26 & 0.11 & $0.05-0.47$ & 2.43 & .015 & 1.84 \\
\hline AoA & 0.03 & 0.01 & $0.00-0.05$ & 2.24 & .026 & 2.42 & -0.30 & 0.12 & $-0.54--0.06$ & -2.45 & .014 & 2.44 \\
\hline Familiarity & -0.04 & 0.01 & $-0.06--0.02$ & -4.19 & $<.001$ & 1.74 & 0.13 & 0.10 & $-0.07-0.33$ & 1.29 & .198 & 1.75 \\
\hline Frequency & -0.02 & 0.01 & $-0.03-0.00$ & -1.61 & .108 & 1.58 & 0.27 & 0.10 & $0.08-0.46$ & 2.78 & .005 & 1.57 \\
\hline OrdCatPos & 0.01 & 0.00 & $0.01-0.02$ & 3.25 & .001 & 3.18 & -0.13 & 0.05 & $-0.24--0.03$ & -2.52 & .011 & 3.14 \\
\hline
\end{tabular}




\begin{tabular}{|c|c|c|c|c|c|c|c|c|c|c|c|c|}
\hline Order & 0.01 & 0.00 & $0.00-0.01$ & 2.16 & .030 & 3.13 & 0.11 & 0.06 & $0.02-0.20$ & 2.43 & .015 & 3.05 \\
\hline NoFeats & -0.02 & 0.01 & $-0.04--0.00$ & -1.99 & .048 & 1.55 & 0.22 & 0.10 & $0.03-0.41$ & 2.24 & .025 & 1.57 \\
\hline IntercorrDens & 0.02 & 0.01 & $-0.00-0.04$ & 1.71 & .088 & 2.20 & -0.25 & 0.11 & $-0.47--0.02$ & -2.17 & .030 & 2.26 \\
\hline NrSemNeigh & 0.01 & 0.01 & $-0.01-0.04$ & 1.02 & .307 & 2.44 & -0.05 & 0.12 & $-0.29-0.18$ & -0.45 & .657 & 2.47 \\
\hline SemSim & 0.01 & 0.01 & $-0.01-0.03$ & 0.90 & .369 & 2.50 & -0.07 & 0.12 & $-0.31-0.17$ & -0.56 & .577 & 2.51 \\
\hline Typicality & 0.00 & 0.01 & $-0.01-0.02$ & 0.37 & .713 & 1.44 & 0.01 & 0.09 & $-0.18-0.19$ & 0.08 & .940 & 1.49 \\
\hline Distinct & 0.03 & 0.01 & $0.01-0.05$ & 2.98 & .003 & 2.04 & -0.15 & 0.11 & $-0.36-0.07$ & -1.32 & .186 & 2.03 \\
\hline \multicolumn{7}{|c|}{ Observations: 20,943} & \multirow{2}{*}{\multicolumn{6}{|c|}{$\begin{array}{l}\text { Observations: } 24,554 \\
\text { Marginal } R^{2} \text { / Conditional } R^{2}: 0.231 / 0.495\end{array}$}} \\
\hline \multicolumn{7}{|c|}{ Marginal $R^{2}$ / Conditional $R^{2}: 0.143$ / 0.456} & & & & & & \\
\hline
\end{tabular}

Note. VIF = Variance Inflation Factor, NameAgr = name agreement, ImageAgr = image agreement, AoA = age of acquisition, OrdCatPos $=$ ordinal

category position, NoFeats $=$ number of semantic features, IntercorrDensity $=$ intercorrelational density, NrSemNeigh $=$ Number of near semantic

neighbours, SemSim = Semantic similarity, Distinct $=$ distinctiveness, Participant $\mid X=$ random slope of $X$ by participants;

values for significant effects $(p<.05)$ are printed in bold. 


\section{Discussion}

Understanding how word-inherent semantic properties of an item affect word production is critical for improving our understanding of the mechanisms and refining theoretical models of spoken word production. However, previous research investigating effects of semantic variables on production has had numerous methodological and statistical limitations, which may have distorted the findings. Here, we overcame these shortcomings and investigated the role of six feature-based semantic variables on the word production process: number of semantic features, intercorrelational density, number of near semantic neighbours, semantic similarity, typicality, and distinctiveness, while controlling for other psycholinguistic variables that have been found to influence word production.

The design of our study also enabled direct comparison with previous literature through a conceptual replication of Rabovsky et al. (2016) that evaluated the reliability of their findings. We therefore investigated effects of number of semantic features and intercorrelational density on word production under reduced (Analysis $1 \mathrm{~A}$ and as in Rabovsky et al.) and subsequently under more adequate control of psycholinguistic variables (Analysis 1B), before investigating the effects of the six semantic variables of interest simultaneously (Analysis 2).

\section{Table 7}

Summary of the findings for semantic variables across all analyses

\begin{tabular}{rcccccccc}
\hline & \multicolumn{2}{c}{$\begin{array}{c}\text { Rabovsky et al. } \\
\text { (2016) }\end{array}$} & \multicolumn{2}{c}{ Analysis 1A } & \multicolumn{2}{c}{ Analysis 1B } & \multicolumn{2}{c}{ Analysis 2 } \\
\cline { 2 - 9 } & latency & accuracy & latency & accuracy & latency & accuracy & latency & accuracy \\
\hline NoFeats & $\nearrow$ & $\nearrow$ & $\varnothing$ & $\nearrow$ & $\varnothing$ & $\nearrow$ & $\nearrow$ & $\nearrow$ \\
IntercorrDens & $\swarrow$ & $\swarrow$ & $\varnothing$ & $\varnothing$ & $\varnothing$ & $\swarrow$ & $\varnothing$ & $\swarrow$ \\
NearSemNeigh & & & & & & & $\varnothing$ & $\varnothing$ \\
SemSim & & & & & & & $\varnothing$ & $\varnothing$ \\
Typicality & & & & & & & $\varnothing$ & $\varnothing$ \\
Distinct & & & & & & $\varnothing$ & $\varnothing$ \\
\hline
\end{tabular}

Note. $\varnothing=$ non-significant effect, $\swarrow=$ poorer performance (slower responses and decreased accuracy with higher values of the semantic variable), $\lambda=$ improved performance (faster responses and higher accuracy with higher values of the semantic variable), blank cells were not investigated, 
NoFeats $=$ number of semantic features, IntcorrDens = intercorrelational density, NearSemNeigh = number of near semantic neighbours, SemSim = semantic similarity, Distinct $=$ distinctiveness.

Table 7 summarises the results of our analyses. Taken together, number of semantic features was the most important and consistent predictor of naming accuracy across analyses and there was also a relatively weak effect on naming latency in the most complex analysis (Analysis 2). Moreover, there were effects of intercorrelational density on naming accuracy (Analyses $1 \mathrm{~B}$ and 2) and an effect of distinctiveness on latency (Analysis 2). Importantly, there was a lack of evidence for effects of number of near semantic neighbours, semantic similarity, or typicality on naming. Below, we first discuss possible reasons for the relatively restricted effects of semantic variables that were found, then we relate our results to those of Rabovsky et al. (2016), and discuss the changes in effects in the different analyses before turning to the theoretical interpretation of individual significant effects.

\section{Limited effects of some semantic variables}

Over and above the effects of number of semantic features and intercorrelational density, as also investigated by Rabovsky et al. (2016), distinctiveness was the only other semantic variable to exert a significant effect on the participants' naming performance (Analysis 2, Tables 6 and 7). That is, there was no evidence that picture naming performance was affected by number of near semantic neighbours, semantic similarity, and typicality.

The absence of effects of these variables is in contrast to most previous work, where significant effects for these semantic variables were reported. This reinforces the validity of the concern, raised in the Introduction, that significant effects in some of the previous studies could have been Type 1 errors, false positives, arising due to a lack of control of other semantic and psycholinguistic variables (see below for more detail). However, particularly for effects of number of semantic neighbours and semantic similarity, differences in significance could also be due to task differences (i.e., speeded vs simple picture naming). Moreover, as evident in Table 1, compared to our study, most previous reports had fewer data points, due to smaller samples of participants and/or 
items, leading to lower statistical power and reliability of the results. In addition, differences in the statistical approach (i.e., taking into account the variability induced by specific participants and items using mixed effects models or disregarding such differences in regression analyses; using semantic variables as continuous or categorical predictors) may have caused differences in the significance of effects of semantic variables. Finally, of course, there is also the very real concern that this literature, as is common, suffers from publication bias towards significant findings.

Importantly, our null effects of the number of near semantic neighbours are in line with previous work using a simple picture naming paradigm (Hameau et al., 2019; Lampe et al., 2017); the studies that found significant effects used a speeded naming paradigm. Similarly, not all previous studies have found significant effects of typicality (Morrison et al., 1992; Woollams, 2012).

\section{Development of effects across analyses}

We now review how the effects of semantic variables changed across the analyses. We do not comment on marginally significant findings and only focus on significant effects $(p<.05)$.

Conceptual replication of Rabovsky et al. (2016). As is summarised in Tables 1 and 7 , Rabovsky et al. (2016) found significant effects of both number of semantic features and intercorrelational density on picture naming latency and accuracy. In Analysis $1 \mathrm{~A}$, where we replicated Rabovsky et al.'s analyses by taking the same semantic and control variables into account, we replicated their facilitatory effect of number of semantic features on naming accuracy, while the effect of intercorrelational density was non-significant. Moreover, we did not replicate Rabovsky et al.'s results for naming latency: Neither number of features nor intercorrelational density showed significant effects on naming latency. However, in the more complex analyses, particularly Analysis 2 that accounted for further control and semantic variables, our findings were broadly consistent with those of Rabovsky et al.: Number of semantic features affected both naming latency and accuracy (facilitatory) and intercorrelational density affected accuracy (inhibitory). Hence, the only effect reported by Rabovsky et al. that failed to reach significance in any of our analyses was the effect of intercorrelational density on naming latency. However, in Rabovsky et al.'s own work this effect also 
did not prove to be robust: While the group effect was significant in Rabovsky et al. (2016), few individual participants were significantly affected, and in Rabovsky et al. (2020) the effect was nonsignificant when the participants first named the pictures.

What could be the cause of the differences between Rabovsky et al.'s (2016) results and those of our Analysis $1 \mathrm{~A}$ ? Given that we had almost three times as many data points as the original study (24,725 data points vs 8,656 data points), our failure to replicate Rabovsky et al. (and other studies reported in Table 1) cannot be due to lack of statistical power relative to the original study; if anything, our findings should be more reliable. The fact that effects that are in line with Rabovsky et al. emerged in our subsequent analyses may suggest that the effects of semantic variables are relatively weak and subject to a certain degree of variability. By including more control variables in the models of Analysis $1 \mathrm{~B}$ and 2 we statistically controlled some of this variability, which resulted in significant effects of more semantic variables (further discussion of this in the next section). Although the difference in the language spoken by the participants (English vs German) may have influenced the results, as the semantic variables are argued to reflect our underlying conceptual representation and lexical processing, there is no reason to expect that these factors should operate differently across languages. However, there are other methodological factors that may have been important: For example, we ensured that name agreement of the experimental items was high for our population, the features were generated in the same language as the experiment, and we used colour photographs, which can improve concept recognition for some items (e.g., lime vs lemon; Bonin et al., 2019; Rossion \& Pourtois, 2004). We would argue that these differences are, once again, likely to make the results of our study more reliable but may also have led to differences in the outcomes of the two studies.

Changes in effects across analyses. As summarised in Table 7, the significance of some semantic variables changed between analyses: The most significant effects of semantic variables were found in the most complex analysis (Analysis 2). Why do more semantic variables effects reach significance with improved statistical control of factors affecting naming? It is well known that (inter-) correlations between variables can cause the effect of one variable to be significant only when another 
variable is not included in the analysis. Moreover, mediator or suppressor variables can also influence the findings, potentially leading to disappearance, overestimation, or underestimation of the effect of an independent variable on the dependent variable (Type 1 or 2 errors) (Hair et al., 2014). The inclusion of such variables in the model can increase the regression coefficient of another variable in the model by removing irrelevant variance from that predictor (e.g., Pandey \& Elliott, 2010)3.

Therefore, it is vital even if a certain influential variable is of no direct interest to the experiment, that it is included in the analysis as the omission of such confounding variables can substantially distort the effects of other variables and lead to spurious results. By keeping effects of independent variables (e.g., psycholinguistic control variables) constant by including them in the statistical analysis, we can study maximally pure contributions of the variables of interest.

In our analyses, most effects were significant under conditions of maximal control (Analysis 2). This suggests that in Analyses $1 \mathrm{~A}$ and $1 \mathrm{~B}$ some effects were underestimated due to uncontrolled suppressor variables or correlations with variables that were not included in the analyses. As explained above, any effects of semantic variables emerging under more rigorous control of other variables can be considered more reliable, because there is less free variance that can, spuriously, be taken up by the variables of interest. Therefore, it seems likely that effects of the semantic variables are rather weak and therefore only able to cross the significance threshold when the variance of other variables is controlled.

\footnotetext{
${ }^{3}$ For example, both familiarity and imageability affect picture naming (higher values making picture naming more accurate and faster) but effects of frequency tend to be stronger. Imagine the situation where in a set of items more familiar words tend to be of lower imageability (there is a negative correlation). If the effects of each variable is examined independently (in a correlation for example), the two variables are in competition. There may be an effect of familiarity, the variable with the strongest effects. However, as the words of higher familiarity are of lower imageability, the effect of imageability will be masked: Familiarity is a suppressor variable. Only when both variables are analysed together and the independent variance of each can be evaluated will a significant effect of imageability become apparent (assuming there is sufficient independent variance).
} 


\section{Theoretical explanations for significant effects}

The effects of the semantic variables that we observed constrain the architecture of word production models, as, to be considered an adequate model of word production, a theory has to be able to account for all these effects. Below we discuss the theoretical explanations of the significant effects found here-effects of number of semantic features, intercorrelational density, and distinctiveness -in the context of current theories of word production.

Number of semantic features. Number of semantic features represented a simple count of the features of a concept according to the McRae et al. (2005) feature database and was the most important predictor of naming accuracy and significant in all three of our analyses. Moreover, in the most complex analysis (Analysis 2), there was a weak effect of number of semantic features on naming latency. Naming was more accurate and faster for words with more semantic features, which is in agreement with previous work by Rabovsky et al. $(2016,2020)$ and Taylor et al. (2012).

Using a neural network model, Rabovsky and McRae (2014; Simulation 2) simulated processing of words with high or low numbers of semantic features. For words with a higher number of semantic features, there was higher semantic activation, corresponding to the summed activation across all semantic features of an item, which Rabovsky and McRae argued could explain facilitatory behavioural effects of number of semantic features. In word production, this increased semantic activation is argued to result in stronger activation of the target word's lexical representation, facilitating its selection and leading to faster and more accurate responses (see also Rabovsky et al., 2016). Current theories of word production would predict facilitated naming of targets with stronger lexical activation (e.g., Abdel Rahman \& Melinger, 2009; Dell, 1986; Howard et al., 2006; Levelt et al., 1999; Oppenheim et al., 2010). Simultaneously, though, a higher number of semantic features may result in the co-activation of a larger number of semantically related lexical representations as these features will be represented in other concepts (e.g., Rabovsky et al., 2016). However, our finding of a facilitatory effect of number of semantic features suggests that any increased lexical competition from co-activated representations (in theories that incorporate competitive mechanisms) is outweighed by 
the conceptual facilitation from the many semantic features. In contrast, concepts with a lower number of semantic features will result in relatively less activation of that concept's lexical representation, with selection therefore being more error prone and slower.

The facilitatory effect from higher numbers of semantic features may also be compatible with other theoretical frameworks: Assuming holistic concepts, the measure of number of semantic features may capture the number of links between a target concept and other concepts. Activation would spread along those links, which ultimately facilitates target processing. Moreover, in the context of an attractor network, concepts with richer semantic representations (e.g., higher numbers of features) build stronger attractor basins, which enable the system to settle faster and more accurately into a stable pattern of activation (Plaut \& Shallice, 1993; see also Pexman et al., 2007).

Intercorrelational Density. Intercorrelational density had an inhibitory effect on naming accuracy, as found previously (Rabovsky et al., 2016, 2020): Participants made significantly more errors on words with higher intercorrelational density. Such words have feature pairs that tend to co-occur across concepts and therefore share a lot of variance. Hence, intercorrelational density measures how strongly the features of a concept cluster together. For example, the features has fur and has four legs also tend to occur with the features has a tail, has ears, etc. (McRae \& Cree, 2002). Features in such intercorrelated clusters have been proposed to boost each other's activation (e.g., via bidirectional links between the features; McRae, 2004; McRae et al., 1997) and simulations (McRae et al., 1997) and previous experimental work (McRae et al., 1999; see also Taylor et al., 2004) have found that correlations between semantic features facilitate conceptual processing. However, in word production, intercorrelational density may also determine the size of the co-activated lexical cohort and the strength of its activation as it reflects the activation that is shared between the target concept and related concepts (Rabovsky et al., 2016).

In McRae et al. (2005) intercorrelational density is calculated as the summed shared variance of a concept's correlated feature pairs. Therefore, higher intercorrelational density can arise in two ways: 1) A concept has many feature pairs that are at least correlated just above threshold $(6.5 \%$ of 
shared variance) or, 2) a concept has at least a few feature pairs that are strongly correlated and share considerable variance. In the first scenario (1), in addition to the target's lexical representation, lexical representations of many other concepts that share the (slightly) correlated semantic features with the target word would be (slightly) co-activated, with the extent of the co-activation depending on the strength of the intercorrelation of their features. It is important to note that in our analyses (but not in all the others in the literature), this is not confounded with the number of semantic features or number of near semantic neighbours as we controlled for these measures. In the second scenario (2), where an item has a small number of strongly correlated pairs, a different pattern emerges with lexical representations of few other concepts receiving activation. However, their co-activation would be stronger due to the stronger correlations among the feature pairs, making them stronger candidates for selection. These examples clearly illustrate that whether a large number of less intercorrelated concepts or a small number of highly intercorrelated concepts are co-activated critically depends on the number of concepts in which the pairs of correlated features occur and on the strength of their correlation. This not only suggests that the consequences of similar intercorrelational density values for lexical activation can vary between items, but that across sets of items the precise effects of intercorrelational density may be unpredictable depending on the balance of scenario 1 and scenario 2 items. In our analyses, given the absence of evidence for an effect of number of near semantic neighbours, it is likely that the stronger co-activation of closely semantically related representations (scenario 2) is the driving force underlying the negative effects of intercorrelational feature density. Similarly, intercorrelational density may represent the strength of the labelled links between a target and other holistic lexical concepts. In that case, spreading activation along those links may cause the co-activation of various concepts and their lexical representations. Irrespective of the type of semantic representation, assuming bidirectional links between conceptual and lexical levels of word production (e.g., Abdel Rahman \& Melinger, 2009; Dell, 1986), co-activated lexical representations will mutually increase their activation. This mutual increase in activation is particularly powerful for coactivated words that are closely related, due to the strength of the correlation of their features. 
Yet, how do these co-activated representations negatively affect the production of the target word? The account outlined above of a negative effect of intercorrelational density on accuracy assumes a theoretical framework incorporating competitive lexical selection: During lexical selection, the co-activated lexical representations compete with the target word for selection. For words with higher intercorrelational density this competition is stronger, particularly when it arises from more closely related co-activated representations (e.g., Abdel Rahman \& Melinger, 2019; Rose \& Abdel Rahman, 2017). The stronger competition negatively impacts the selection of the target's lexical representation, leading to increased chances of incorrectly selecting a co-activated representation, which can ultimately cause more naming errors. The precise mechanism underlying this competition is still unclear and different ways have been suggested to implement it (e.g., Luce choice mechanism, e.g., Luce, 1959; Abdel Rahman \& Melinger, 2009, 2019; Levelt et al., 1999; Roelofs, 1997; lateral inhibitory links between active representations at the lexical level, e.g., Howard et al., 2006; Caramazza, 1997; McClelland \& Rumelhart, 1981). However, regardless of the details of the competitive mechanism, the presence of more or more strongly co-activated representations will result in poorer performance.

On the other hand, Oppenheim et al.'s (2010) learning mechanism may also be able to explain the poorer performance on items with higher intercorrelational density. According to Oppenheim et al., interference is due to implicit incremental learning, which causes weakening of the connections between the semantic representation of the target and the lexical representations of co-activated but unnamed alternatives after retrieval of the target word. Each act of lexical retrieval results in persistent learning that adjusts the weights of the connections between semantic and lexical representations. Importantly, in our analyses we have equated the influence of other factors, like frequency, between words of lower and higher intercorrelational density. Thus, in this scenario, across a lifetime, words of lower and higher intercorrelational density will be selected at a comparable rate. Consequently, strengthening of conceptual to lexical connections after successful selection would apply equally to words of lower and higher intercorrelational density. However, it seems possible that, everything else 
being equal, words with higher intercorrelational density would be co-activated more often via their strongly intercorrelated features, in contrast to words with lower intercorrelational density. When these items are co-activated, but not selected, the connections between their semantic and lexical representations would be increasingly and persistently weakened. When a word with such weakened connections becomes the target in the experiment, its lexical representation is harder to access, resulting in relatively poorer performance. In sum, although computational modelling may be needed to test this prediction, Oppenheim et al.'s theoretical framework also seems to be able to account for our finding of an inhibitory effect of intercorrelational density.

Finally, inhibitory effects of intercorrelational density on naming accuracy could also be explained in Dell's (1986) theory where the most active lexical representation is selected, irrespective of co-activated lexical representations. In the interactive architecture, the bi-directional information flow between the semantic and the lexical levels could be particularly strong amongst strongly intercorrelated semantic features, leading to stronger activation of non-target lexical representations, which can erroneously exceed the target word's activation and therefore be selected. This would increase the number of naming errors for words with higher intercorrelational density, as reported here.

Distinctiveness. Distinctiveness was defined as the mean uniqueness of the features of a concept in the feature norm database, with concepts with higher distinctiveness, by definition, having more unique and less shared features. The effect of distinctiveness was inhibitory ${ }^{4}$, which is in contrast to previous studies that have investigated an effect of concept distinctiveness in picture naming: Rabovsky et al. (2016) and Taylor et al. (2012) found faster and more accurate responses for words with higher distinctiveness (see also Humphreys et al., 1988, for a facilitatory effect of a similar measure). As an explanation for facilitatory effects of distinctiveness, distinctive features have been suggested to play a privileged role in conceptual processing (e.g., Cree et al., 2006; Taylor et al., 2012),

\footnotetext{
${ }^{4}$ The effect was also inhibitory in an exploratory, not preregistered, analysis in which intercorrelational density was not included in the analysis, following Rabovsky et al. (2016) (please refer to the Introduction for details on the analysis conducted by Rabovsky et al.).
} 
leading to faster and stronger activation of such features and to stronger activation of the respective concept in feature verification tasks $5^{5}$.

If distinctive features are preferentially processed at the conceptual level, how can our finding of a negative effect of higher distinctiveness on naming be explained? When processing a word with higher distinctiveness, due to its rather unique semantic features, only a few semantically related lexical representations will be co-activated via the features they share with the target word. Alternatively, in a holistic semantic architecture, there are (holistic) concepts which represent features (e.g., seeds), which are connected to a range of other concepts (e.g., apple, grapes, grass).

Distinctiveness of a target (e.g., apple) could capture the average number of these connections for each of the (feature) concepts to which the target is connected (e.g., red, seeds). For words with fewer connections (i.e., words with higher distinctiveness), target processing would result in reduced activation spreading to other holistic lexical concepts. Thus, irrespective of the semantic architecture, enhanced co-activation and thus competition for lexical selection would be expected for words with lower distinctiveness, which predominantly have features that are shared with other concepts (see Vieth et al., 2014 Figure 1, for a visualisation) or may be connected to many other concepts, which are highly connected themselves. Thus, our inhibitory effect of higher distinctiveness is unlikely to be due to stronger competition between co-activated (lexical) representations, as fewer competing candidates are expected to be co-active when processing words with higher distinctiveness.

Importantly, this account of competition from strongly co-activated representations is also heavily based on the notion of the number of near semantic neighbours (i.e., words that share many semantic features with the target word) competing with the target for selection. Yet, in our analyses, and in contrast to the previous studies by Rabovsky et al. (2016) and Taylor et al. (2012), effects of the number of near semantic neighbours were accounted for and held constant by including this measure in the analysis. Thus, our statistical approach enabled us to obtain a more precise (purer) estimate of

\footnotetext{
${ }^{5}$ Cree et al. (2006) explained the facilitatory effect of distinctiveness in the context of an attractor network: The strength and speed of activation of privileged distinctive features allows the network to enter the correct attractor basin faster, which enables it to settle in a stable state more rapidly.
} 
the effect of mean concept distinctiveness on naming, over and above number of near semantic neighbours, to evaluate the effect of a concept having distinctive features.

Critically, it has not yet been fully established exactly how numerous or closely related to the target co-activated lexical representations must be to compete for selection; however, there is research suggesting that more closely related representations cause stronger competition (e.g., Rose et al., 2019). Thus, while competition from many co-activated competitors is unlikely to be the mechanism underlying the inhibitory effect of distinctiveness, it may be possible that the effect could be caused by few highly related co-activated representations. There may, for example, be only one or two concepts that are closely related to the target, sharing many of its relatively unique features, and thus acting as strong competitors for selection, causing the observed inhibitory effect. In future studies, this could perhaps be tested by operationalising the semantic similarity between the target and its closest semantic neighbour(s) as a further semantic variable.

Similarly, in Oppenheim et al.'s (2010) framework we would expect an advantage for words that have higher distinctiveness, through weakening of connections between shared features and non-target lexical representations. Every time a lexical item is retrieved, when there are shared features, links from those features to non-target representations would be subject to increased weakening. A word with lower distinctiveness (which has more shared features) would be subject to this weakening to a higher degree than a word with higher distinctiveness, resulting in poorer performance on items of lower distinctiveness and not of higher distinctiveness as we found in our data.

In contrast to the accounts of a privileged status of distinctive features described above, the Conceptual Structure Account (e.g., Tyler et al., 2000; Tyler \& Moss, 2001) of semantic memory argues that there is a processing disadvantage for distinctive features of living things and an advantage for shared features, while there is no such dramatic difference for artifacts. The disadvantage for distinctive features of living things is thought to arise because they are only weakly correlated with other features and hence do not benefit from mutual activation among correlated features. This 
causes slowed access to such features and makes them more vulnerable to loss in impaired semantic processing. In contrast, distinctive features of artifacts are correlated more strongly with other features and are therefore thought to benefit from mutual activation among the correlated features. Hence, this account predicts an inhibitory effect of distinctiveness for living things, which was found in experimental work using feature verification and definition tasks with individuals with semantic impairment (Moss et al., 1998, 2002) and unimpaired participants (Randall et al., 2004). Randall et al. found the predicted advantage in feature verification for shared over distinctive features for living things (while there was no robust main effect of distinctiveness across domains on feature verification speed or accuracy). Moreover, distinctive features of living things were verified more slowly than distinctive features of non-living things and higher error rates were reported for distinctive rather than for shared features when this data was computationally simulated. However, Cree et al. (2006) pointed out several shortcomings in Randall et al.'s work (e.g., insufficient matching), and reported findings that were inconsistent with Randall et al.: Effects of distinctiveness that were comparable for living and nonliving things.

While the account by Randall et al. (2004) predicts an inhibitory effect for distinctiveness for some items (i.e., living things), it also relies heavily on intercorrelations between features to explain the effects. To determine whether the inhibitory effect in our data was particularly strong for living things, as predicted by the Conceptual Structure Account, we ran exploratory post-hoc analyses ${ }^{6}$ of the naming latency and accuracy data that included an interaction between distinctiveness and animacy (reported in Appendix F). While the interaction was non-significant for naming accuracy, it was significant for the naming latency analysis. However, the findings were in the opposite direction to that predicted by the Conceptual Structure Account: Responses to living things were unaffected by distinctiveness, but there was a significant inhibitory effect for non-living things. Hence, while the Conceptual Structure Account is consistent with the general direction of our inhibitory finding for

\footnotetext{
${ }^{6}$ As these were unplanned post-hoc analyses to explore a particular finding in more detail, these analyses were not preregistered.
} 
distinctiveness, its more specific predictions do not hold for our data. Importantly, the Conceptual Structure Account and most previous investigations of distinctiveness were designed in the context of category specific impairments and the processing of single features, which makes it hard to generalise the frameworks and findings to our work. Moreover, in contrast to the current study, many previous studies did not test for an effect of overall concept distinctiveness and did not investigate picture naming.

In our study, we held both intercorrelational density and number of near semantic neighbours constant and still found a significant inhibitory effect of distinctiveness. As the origin of our effect and its theoretical interpretation are unclear, we suggest that future computational modelling could be helpful for uncovering the mechanisms underlying this effect and lead to better understanding of it.

\section{Contributions and limitations of this research}

In this study, we determined feature-based semantic variables that are important for word production. Our findings of effects of semantic variables allowed us to critique theories of spoken word production and to evaluate how they must operate to be able to explain the results. Interestingly, we identified semantic variables that had both facilitatory and inhibitory influences on picture naming. Thus, theories of word production need mechanisms that can account for both effects. While, as detailed in the previous sections, semantic facilitation is implemented in current theories as spreading activation or feedback from lexical to semantic representations, interference can be explained via lexical competition or could be due to long-term adjustments of conceptual to lexical connections.

Importantly, current theories of word production are underspecified regarding the way semantic variables may affect performance. Of the currently available theories of word production, Abdel Rahman and Melinger $(2009,2019)$ explicitly address word-inherent variables and their Swinging Lexical Network Hypothesis comprises the architectural elements (i.e., spreading activation and lexical competition) to parsimoniously explain both facilitatory and inhibitory effects of such variables. Other theories (e.g., Dell, 1986; Oppenheim et al., 2010) were able to only account for some 
of our results. Crucially, for current theories to explain the findings, we were required to make additional assumptions regarding processing or the encoding of semantic relations that are not explicitly specified in the theories. Thus, the current theories of word production need further modification and specification to explain effects of semantic variables.

Important methodological contributions of this paper have been highlighted throughout this section: the importance of rigorous control of other variables influencing word production (i.e., both control and other semantic variables), highly powered statistical analyses, and considering variables of interest simultaneously.

This study was also subject to some limitations. While we showed that multicollinearity between the measures included in our analyses did not occur at compromising levels, there were some relatively high correlations between semantic variables (Table 3). However, using other statistical techniques (e.g., Principal Component Analysis) was neither suitable for addressing our research goals, nor possible given a single measure of each variable. In addition, according with our study's aim to identify individual semantic variables that affect picture naming performance, in the sections above, we assessed how the effect of each of the significant semantic variables could be explained in the context of current theories of word production. While we covered a range of semantic variables that capture different aspects of the flow of activation at the semantic level and co-activation at the lexical level, it may also be the case that there are other (potentially composite) measures that outperform the variables used here in capturing the dynamics of activation spread and lexical co-activation. Further research and modelling could address this.

\section{Use of semantic feature norms}

We chose which semantic variables to study based on whether it was possible to quantify these variables objectively rather than using ratings. We chose variables that could be operationalised based on the information provided in semantic feature databases (and specifically that of McRae et al., 2005). However, in theories of word production there is no theoretical agreement that conceptual representations are actually decomposed into semantic features: While some accounts assume 
decomposed conceptual representations (e.g., Dell, 1986; Oppenheim et al., 2010), others presume non-decomposed, holistic, representations of meaning (e.g., Levelt et al., 1999; Roelofs, 1992).

While we use the context of semantic features to characterise aspects of the semantic representation of concepts and relationships between concepts, and to interpret our findings, this does not imply that we favour theories of decomposed conceptual representations over nondecomposed theories. The semantic variables we studied here could also reflect semantic relationships as described by non-decomposed theories of semantics such as direct connections between concepts (Collins \& Loftus, 1975) or indirect connections via property nodes (Collins $\&$ Quillian, 1969). Throughout the theoretical explanations of our significant findings, we pointed towards possible ways the significant variables may operate in a holistic semantic architecture. Importantly though, computational modelling may be helpful to further assess our hypotheses concerning the mechanisms underlying the effects of semantic variables in the context of different semantic architectures and to aid the interpretation of the effects of these variables.

Hence, while the effects of this study can more readily be explained in the context of models assuming feature-based semantic representations, our findings do not allow us to adjudicate between decomposed and non-decomposed semantic representations.

Participant generated semantic features are a verbalisation and temporary abstraction of complex semantic representations, which have developed through multisensory experience with the concept in real life (e.g., McRae, 2004). While semantic feature databases provide one of the best currently available approaches to specifying conceptual content, researchers have also pointed out some limitations (e.g., Amsel \& Cree, 2013; Randall et al., 2004). While operationalising our semantic variables based on a semantic feature database (McRae et al., 2005), we accept the shortcomings of this resource, such as, for example, the underrepresentation of highly frequent features (e.g., breathes) or the absence of features that are difficult to verbalise (Cree \& McRae, 2003).

However, in contrast to ratings, calculating semantic variables based on semantic features represents a more objective way of operationalising conceptual knowledge, as participants are not 
asked to directly rate a certain aspect of the semantic representation (e.g., concept typicality within its semantic category). Nevertheless, while measures based on semantic feature databases are transparent and reproducible, their calculation is based on the rather limited number of items in the corpus.

As described in the Introduction, semantic feature databases present only one way of describing conceptual knowledge. Semantic relationships and similarity could also be objectively captured by other semantic dimensions, such as associations (e.g., De Deyne et al., 2019; Nelson et al., 2004) or contextual co-occurences (e.g., Latent Semantic Analysis (LSA), Landauer et al., 1998; Continuous bag-of-words model (CBOW), Mikolov et al., 2013; Global Vectors for word representation (GloVe), Pennington et al., 2014). In this study, we conducted a thorough investigation of the featurebased dimension of semantic relationships as previous research suggested a large variety of featurebased variables that affected word production. In addition, when comparing different measures of semantic neighbourhood density, Hameau et al. (2019) found a feature-based measure to be a better predictor of picture naming in people with aphasia than association-based or contextual semantic neighbourhood density. Future research could extend our approach by including semantic variables derived from other semantic dimensions to determine the extent to which contextual and associationbased similarity measures can also affect word production. Such an approach would also allow assessment of how far the observed dynamics of semantic and lexical activation in word production depend on the structural assumptions of semantic feature databases, and, finally, to identify the dimension of semantics that captures most of the variability in our data.

\section{Conclusion}

We have reported an investigation of effects of six feature-based semantic variables (number of semantic features, intercorrelational density, number of near semantic neighbours, semantic similarity, typicality, and distinctiveness) on the picture naming performance of a large group of young English speakers. We found that number of semantic features was the most important predictor of naming accuracy and, to a lesser extent, of naming latency, with further effects of intercorrelational 
density and distinctiveness. These semantic variables should therefore be considered in further research.

This research allowed us to determine which variables influence behaviour and develop hypotheses regarding the mechanisms and dynamics underpinning these effects in theories of spoken word production. The findings of our study indicate that both aspects of the richness of the semantic representation and of relationships between concepts play an important role in semantic and lexical processing during word production. Importantly though, some previously reported effects of several semantic variables (namely number of near semantic neighbours and semantic similarity (in speeded naming), typicality, and the direction of the effect of distinctiveness) did not replicate here, which highlights once again the importance of a well-controlled study design, to minimise the risk of miscalibration in estimating such effects. 


\section{References}

Abdel Rahman, R., \& Melinger, A. (2009). Semantic context effects in language production: A swinging lexical network proposal and a review. Language and Cognitive Processes, 24(5), 713-734. https://doi.org/10.1080/01690960802597250

Abdel Rahman, R., \& Melinger, A. (2019). Semantic processing during language production: An update of the swinging lexical network. Language, Cognition and Neuroscience, 34(9), 1176-1192. https://doi.org/10.1080/23273798.2019.1599970

Alario, F.-X., Ferrand, L., Laganaro, M., New, B., Frauenfelder, U. H., \& Segui, J. (2004). Predictors of picture naming speed. Behavior Research Methods, Instruments, \& Computers, 36(1), 140-155. https://doi.org/10.3758/BF03195559

Allison, P. (2012). When can you safely ignore multicollinearity? Statistical Horizons. https://statisticalhorizons.com/multicollinearity

Amsel, B. D., \& Cree, G. S. (2013). Semantic richness, concreteness, and object domain: An electrophysiological study. Canadian Journal of Experimental Psychology, 67(2), 117-129. https://doi.org/10.1037/a0029807

Baayen, H. R., \& Milin, P. (2010). Analyzing reaction times. International Journal of Psychological Research, 3(2), 12-28. https://doi.org/10.21500/20112084.807

Bates, D., Kliegl, R., Vasishth, S., \& Baayen, H. (2015). Parsimonious mixed models. ArXiv, 1-27. https://doi.org/arXiv:1506.04967

Bates, D., Maechler, M., Bolker, B., Walker, S., Christensen, R. H. B., Singmann, H., Dai, B., Scheipl, F., Grothendieck, G., \& Green, P. (2017). Ime4: Linear Mixed-Effects Models using "Eigen" and S4 (R package version 1.1-21).

Bock, K., \& Levelt, W. (1994). Language production: Grammatical encoding. In M. A. Gernsbacher (Ed.), Handbook of psycholinguistics (pp. 945-984). Academic Press. https://doi.org/10.3115/981923.981940

Boersma, P., \& Weenink, D. (2019). Praat: Doing phonetics by computer (Version 6.0.49). 
https://doi.org/http://www.praat.org/

Bonin, P., Méot, A., Laroche, B., Bugaiska, A., \& Perret, C. (2019). The impact of image characteristics on written naming in adults. Reading and Writing, 32(1), 13-31. https://doi.org/10.1007/s11145$017-9727-3$

Bormann, T. (2011). The role of lexical-semantic neighborhood in object naming: Implications for models of lexical access. Frontiers in Psychology, 2, 1-11.

https://doi.org/10.3389/fpsyg.2011.00127

Caramazza, A. (1997). How many levels of processing are there in lexical access? Cognitive Neuropsychology, 14(1), 177-208. https://doi.org/10.1080/026432997381664

Collins, A. M., \& Loftus, E. F. (1975). A spreading-activation theory of semantic processing. Psychological Review, 82(6), 407-428. https://doi.org/10.1037/0033-295X.82.6.407

Collins, A. M., \& Quillian, M. R. (1969). Retrieval time from semantic memory. Journal of Verbal Learning and Verbal Behavior, 8(2), 240-247. https://doi.org/https://doi.org/10.1016/S00225371(69)80069-1

Cortese, M. J., \& Fugett, A. (2004). Imageability ratings for 3,000 monosyllabic words. Behavior Research Methods, Instruments, \& Computers, 36(3), 384-387.

https://doi.org/10.3758/BF03195585

Cree, G. S., McNorgan, C., \& McRae, K. (2006). Distinctive features hold a priviledged status in the computation of word meaning: Implications for theories of semantic memory. Journal of Experimental Psychological Learning, Memory and Cognition, 32(4), 643-658.

https://doi.org/10.1037/0278-7393.32.4.643

Cree, G. S., \& McRae, K. (2003). Analyzing the factors underlying the structure and computation of the meaning of chipmunk, cherry, chisel, cheese, and cello (and many other such concrete nouns). Journal of Experimental Psychology: General, 132(2), 163-201. https://doi.org/10.1037/00963445.132.2.163

Davis, C. J. (2005). N-watch: A program for deriving neighborhood size and other psycholinguistic 
statistics. Behavior Research Methods, 3711), 65-70. https://doi.org/10.3758/BF03206399

De Deyne, S., Navarro, D. J., Perfors, A., Brysbaert, M., \& Storms, G. (2019). The "Small World of Words" English word association norms for over 12,000 cue words. Behavior Research Methods, 51(3), 987-1006. https://doi.org/10.3758/s13428-018-1115-7

Dell'Acqua, R., Lotto, L., \& Job, R. (2000). Naming times and standardized norms for the Italian PD/DPSS set of 266 pictures: Direct comparisons with American, English, French, and Spanish published databases. Behavior Research Methods, Instruments, \& Computers, 32(4), 588-615. https://doi.org/10.3758/BF03200832

Dell, G. S. (1986). A spreading-activation theory of retrieval in sentence production. Psychological Review, 93(3), 283-321. https://doi.org/10.1037/0033-295X.93.3.283

Devereux, B. J., Tyler, L. K., Geertzen, J., \& Randall, B. (2014). The Centre for Speech, Language and the Brain (CSLB) concept property norms. Behavior Research Methods, 46(4), 1119-1127. https://doi.org/10.3758/s13428-013-0420-4

Ewald, A., Aristei, S., Nolte, G., \& Rahman, R. A. (2012). Brain oscillations and functional connectivity during overt language production. Frontiers in Psychology, 3, 1-12. https://doi.org/10.3389/fpsyg.2012.00166

Fieder, N., Wartenburger, I., \& Abdel Rahman, R. (2019). A close call: Interference from semantic neighbourhood density and similarity in language production. Memory \& Cognition, 47(1), 145168. https://doi.org/10.3758/s13421-018-0856-y

Fujihara, N., Nageishi, Y., Koyama, S., \& Nakajima, Y. (1998). Electrophysiological evidence for the typicality effect of human cognitive categorization. International Journal of Psychophysiology, 29(1), 65-75. https://doi.org/10.1016/S0167-8760(97)00099-8

Garrard, P., Lambon Ralph, M. A., Patterson, K., Pratt, K. H., \& Hodges, J. R. (2005). Semantic feature knowledge and picture naming in dementia of Alzheimer's type: A new approach. Brain and Language, 93(1), 79-94. https://doi.org/10.1016/j.bandl.2004.08.003

Gauvin, H. S., Jonen, M. K., Choi, J., McMahon, K., \& de Zubicaray, G. I. (2018). No lexical competition 
without priming: Evidence from the picture-word interference paradigm. Quarterly Journal of Experimental Psychology, 71(12), 2562-2570. https://doi.org/10.1177/1747021817747266

Gilhooly, K. J., \& Logie, R. H. (1980). Age-of-acquisition, imagery, concreteness, familiarity, and ambiguity measures for 1,944 words. Behavior Research Methods \& Instrumentation, 12(4), 395427. https://doi.org/10.3758/BF03201693

Grossman, M., Robinson, K., Biassou, N., White-Devine, T., \& D'Esposito, M. (1998). Semantic memory in Alzheimer's disease: Representativeness, ontologic category, and material. Neuropsychology, 12(1), 34-42. https://doi.org/10.1037/0894-4105.12.1.34

Hair, J. F., Black, W. C., Babin, B. J., \& Anderson, R. E. (2014). Multivariate data analysis (7th ed.). Pearson.

Hameau, S., Nickels, L., \& Biedermann, B. (2019). Effects of semantic neighbourhood density on spoken word production. Quarterly Journal of Experimental Psychology, 72(12), 2752-2775. https://doi.org/10.1177/1747021819859850

Holmes, S. J., \& Ellis, A. W. (2006). Age of acquisition and typicality effects in three object processing tasks. Visual Cognition, 13(7-8), 884-910. https://doi.org/10.1080/13506280544000093

Howard, D., Nickels, L., Coltheart, M., \& Cole-Virtue, J. (2006). Cumulative semantic inhibition in picture naming: Experimental and computational studies. Cognition, 100(3), 464-482. https://doi.org/10.1016/j.cognition.2005.02.006

Humphreys, G. W., Riddoch, M. J., \& Quinlan, P. T. (1988). Cascade processes in picture identification. Cognitive Neuropsychology, 5(1), 67-104. https://doi.org/10.1080/02643298808252927

Hutcheson, G., \& Sofroniou, N. (1999). The multivariate social scientist: Introductory statistics using generalized linear models. Sage Publications.

Johnston, R. A., Dent, K., Humphreys, G. W., \& Barry, C. (2010). British-English norms and naming times for a set of 539 pictures: The role of age of acquisition. Behavior Research Methods, 42(2), 461469. https://doi.org/10.3758/BRM.42.2.461

Jolicoeur, P., Gluck, M. A., \& Kosslyn, S. M. (1984). Pictures and names: Making the connection. 
Cognitive Psychology, 16(2), 243-275. https://doi.org/10.1016/0010-0285(84)90009-4

Kremin, H. (1986). Spared naming without comprehension. Journal of Neurolinguistics, 2(1-2), 131150. https://doi.org/10.1016/S0911-6044(86)80008-2

Kuznetsova, A., Brockhoff, P. B., \& Christensen, R. H. B. (2017). ImerTest package: Tests in linear mixed effects models. Journal of Statistical Software, 82(13), 1-26. https://doi.org/10.18637/jss.v082.i13 Lampe, L. F., Bürki, A., Sowman, P. F., Hameau, S., \& Nickels, L. (2021). Effects of semantic variables on processes during word planning for production: Evidence from electrophysiological data. Manuscript in Preparation.

Lampe, L. F., Fieder, N., Krajenbrink, T., \& Nickels, L. (2017). Semantische Nachbarschaft in der Wortproduktion bei Aphasie [Semantic neighbourhood in word production in aphasia]. In A. Adelt, Ö. Yetim, C. Otto, \& T. Fritzsche (Eds.), Spektrum Patholinguistik - Panorama Patholinguistik: Sprachwissenschaft trifft Sprachtherapie (Vol. 10, pp. 103-114). Universitätsverlag Potsdam. https://publishup.uni-potsdam.de/opus4-ubp/frontdoor/index/index/docld/39701

Lampe, L. F., Hameau, S., Bürki, A., Fieder, N., Sowman, P. F., \& Nickels, L. (2019). Investigating effects of semantic variables on word production. Preregistration on the Open Science Framework. osf.io/yw6ma

Lampe, L. F., Hameau, S., \& Nickels, L. (2021). Are they really stronger? Comparing effects of semantic variables in speeded deadline and standard picture naming. Manuscript Submitted for Publication.

Landauer, T. K., Foltz, P. W., \& Laham, D. (1998). An introduction to latent semantic analysis. Discourse Processes, 25(2-3), 259-284. https://doi.org/10.1080/01638539809545028

Levelt, W. J. M., Roelofs, A., \& Meyer, A. S. (1999). A theory of lexical access in speech production. Behavioral and Brain Sciences, 22(1), 1-38. https://doi.org/10.1017/S0140525X99001776

Luce, R. D. (1959). Individual choice behavior: A theoretical analysis. Wiley.

Lüdecke, D. (2019). sjPlot: Data visualization for statistics in social science (R package version 2.6.3). https://cran.r-project.org/package=sjPlot 
Mahon, B. Z., Costa, A., Peterson, R., Vargas, K. A., \& Caramazza, A. (2007). Lexical selection is not by competition: A reinterpretation of semantic interference and facilitation effects in the pictureword interference paradigm. Journal of Experimental Psychology: Learning, Memory, and Cognition, 33(3), 503-535. https://doi.org/10.1037/0278-7393.33.3.503

McClelland, J. L., \& Rumelhart, D. E. (1981). An interactive activation model of context effects in letter perception: Part I. An account of basic findings. Psychological Review, 88(5), 375-407. https://doi.org/10.1037/0033-295X.88.5.375

McRae, K. (2004). Semantic memory: Some insights from feature-based connectionist attractor networks. In B. H. Ross (Ed.), The psychology of learning and motivation - Advances in research and theory (pp. 41-86). Elsevier Academic Press. https://doi.org/10.1016/S0079-7421(03)45002-0

McRae, K., \& Cree, G. S. (2002). Factors underlying category-specific impairments. In E. M. E. Forde \& G. W. Humphreys (Eds.), Category specificity in brain and mind (pp. 211-249). Psychology Press. McRae, K., Cree, G. S., Seidenberg, M. S., \& McNorgan, C. (2005). Semantic feature production norms for a large set of living and nonliving things. Behavior Research Methods, 37(4), 547-559. https://doi.org/10.3758/BF03192726

McRae, K., Cree, G. S., Westmacott, R., \& Sa, V. R. De. (1999). Further evidence for feature correlations in semantic memory. Canadian Journal of Experimental Psychology, 53(4), 360-373. https://doi.org/10.1037/h0087323

McRae, K., de Sa, V. R., \& Seidenberg, M. S. (1997). On the nature and scope of featural representations of word meaning. Journal of Experimental Psychology: General, 126(2), 99-130. https://doi.org/10.1080/10430719008404646

Melinger, A., \& Abdel Rahman, R. (2013). Lexical selection is competitive: Evidence from indirectly activated semantic associates during picture naming. Journal of Experimental Psychology: Learning, Memory, and Cognition, 39(2), 348-364. https://doi.org/10.1037/a0028941

Mikolov, T., Chen, K., Corrado, G., \& Dean, J. (2013). Efficient estimation of word representations in vector space. 1st International Conference on Learning Representations (ICLR), 1-12. 
https://arxiv.org/abs/1301.3781

Millard, S. P. (2013). EnvStats: An R package for environmental statistics. Springer.

Miozzo, M., Pulvermüller, F., \& Hauk, O. (2015). Early parallel activation of semantics and phonology in picture naming: Evidence from a multiple linear regression MEG study. Cerebral Cortex, 25(10), 3343-3355. https://doi.org/10.1093/cercor/bhu137

Mirman, D. (2011). Effects of near and distant semantic neighbors on word production. Cognitive, Affective and Behavioral Neuroscience, 11(1), 32-43. https://doi.org/10.3758/s13415-010-0009-7

Mirman, D., \& Graziano, K. M. (2013). The neural basis of inhibitory effects of semantic and phonological neighbors in spoken word production. Journal of Cognitive Neuroscience, 25(9), 1504-1516. https://doi.org/10.1162/jocn_a_00408

Mirman, D., \& Magnuson, J. S. (2008). Attractor dynamics and semantic neighborhood density: Processing is slowed by near neighbors and speeded by distant neighbors. Journal of Experimental Psychology: Learning, Memory, and Cognition, 34(1), 65-79. https://doi.org/10.1037/0278-7393.34.1.65

Morrison, C. M., Ellis, A. W., \& Quinlan, P. T. (1992). Age of acquisition, not word frequency, affects object naming, not object recognition. Memory \& Cognition, 2066), 705-714. https://doi.org/10.3758/BF03202720

Moss, H. E., Tyler, L. K., \& Devlin, J. T. (2002). The emergence of category specific deficits in a distributed semantic system. In E. Forde \& G. W. Humphreys (Eds.), Category-specificity in brain and mind (pp. 115-145). Psychology Press.

Moss, H. E., Tyler, L. K., Durrant-Peatfield, M., \& Bunn, E. M. (1998). 'Two eyes of a see-through': Impaired and intact semantic knowledge in a case of selective deficit for living things. Neurocase, 4(4-5), 291-310. https://doi.org/10.1080/13554799808410629

Nelson, D. L., McEvoy, C. L., \& Schreiber, T. A. (2004). The University of South Florida free association, rhyme, and word fragment norms. Behavior Research Methods, Instruments, \& Computers, 36(3), 402-407. https://doi.org/10.3758/BF03195588 
Oppenheim, G. M., Dell, G. S., \& Schwartz, M. F. (2010). The dark side of incremental learning: A model of cumulative semantic interference during lexical access in speech production. Cognition, 114(2), 227-252. https://doi.org/10.1016/j.cognition.2009.09.007

Paivio, A., Yuille, J. C., \& Madigan, S. A. (1968). Concreteness, imagery, and meaningfulness values for 925 nouns. Journal of Experimental Psychology, 76(1, Pt.2), 1-25. https://doi.org/10.1037/h0025327

Pandey, S., \& Elliott, W. (2010). Suppressor variables in social work research: Ways to identify in multiple regression models. Journal of the Society for Social Work and Research, 1(1), 28-40. https://doi.org/10.5243/jsswr.2010.2

Pennington, J., Socher, R., \& Manning, C. (2014). GloVe: Global vectors for word representation. Conference on Empirical Methods in Natural Language Processing (EMNLP), 1532-1543. https://doi.org/10.3115/v1/D14-1162

Perret, C., \& Bonin, P. (2019). Which variables should be controlled for to investigate picture naming in adults? A Bayesian meta-analysis. Behavior Research Methods, 51(6), 2533-2545. https://doi.org/10.3758/s13428-018-1100-1

Pexman, P. M., Hargreaves, I. S., Edwards, J. D., Henry, L. C., \& Goodyear, B. G. (2007). The neural consequences of semantic richness: When more comes to mind, less activation is observed. Psychological Science, 18(5), 401-406. https://doi.org/10.1111/j.1467-9280.2007.01913.x Pexman, P. M., Holyk, G. G., \& Monfils, M.-H. (2003). Number-of-features effects and semantic processing. Memory \& Cognition, 31(6), 842-855. https://doi.org/10.3758/BF03196439

Pexman, P. M., Siakaluk, P. D., \& Yap, M. J. (2013). Introduction to the research topic meaning in mind: Semantic richness effects in language processing. Frontiers in Human Neuroscience, 7, 1-3. https://doi.org/10.3389/fnhum.2013.00723

Plaut, D. C., \& Shallice, T. (1993). Deep dyslexia: A case study of connectionist neuropsychology. Cognitive Neuropsychology, 195), 377-500. https://doi.org/10.1080/02643299308253469

Python, G., Fargier, R., \& Laganaro, M. (2018). ERP evidence of distinct processes underlying semantic 
facilitation and interference in word production. Cortex, 99, 1-12.

https://doi.org/10.1016/j.cortex.2017.09.008

Qualtrics. (2018). Qualtrics (Version 04/18). https://doi.org/https://www.qualtrics.com

R Core Team. (2018). R: A language and environment for statistical computing. R Foundation for Statistical Computing. https://www.r-project.org/

Rabovsky, M., \& McRae, K. (2014). Simulating the N400 ERP component as semantic network error: Insights from a feature-based connectionist attractor model of word meaning. Cognition, 132(1), 68-89. https://doi.org/10.1016/j.cognition.2014.03.010

Rabovsky, M., Schad, D. J., \& Abdel Rahman, R. (2016). Language production is facilitated by semantic richness but inhibited by semantic density: Evidence from picture naming. Cognition, 146, 240244. https://doi.org/10.1016/j.cognition.2015.09.016

Rabovsky, M., Schad, D. J., \& Abdel Rahman, R. (2020). Semantic richness and density effects on language production: Electrophysiological and behavioral evidence. Journal of Experimental Psychology: Learning, Memory, and Cognition, Advance online publication. https://doi.org/10.1037/xIm0000940

Randall, B., Moss, H. E., Rodd, J. M., Greer, M., \& Tyler, L. K. (2004). Distinctiveness and correlation in conceptual structure: Behavioral and computational studies. Journal of Experimental Psychology: Learning, Memory, and Cognition, 302), 393-406. https://doi.org/10.1037/0278-7393.30.2.393

Rico Duarte, L., \& Robert, C. (2014). Semantic richness influences naming pictures in old age and in Alzheimer's disease. Journal of Neurolinguistics, 31, 55-68.

https://doi.org/10.1016/j.jneuroling.2014.06.004

Roelofs, A. (1992). A spreading-activation theory of lemma retrieval in speaking. Cognition, 42, 107142. https://doi.org/10.1016/0010-0277(92)90041-F

Roelofs, A. (1997). A case for nondecomposition in conceptually driven word retrieval. Journal of Psycholinguistic Research, 26(1), 33-67. https://doi.org/10.1023/A:1025060104569

Rogers, T. T., Patterson, K., Jefferies, E., \& Lambon Ralph, M. A. (2015). Disorders of representation and 
control in semantic cognition: Effects of familiarity, typicality, and specificity. Neuropsychologia, 76, 220-239. https://doi.org/10.1016/j.neuropsychologia.2015.04.015

Rogerson, P. (2011). Statistical methods for geography. SAGE Publications, Ltd. https://doi.org/10.4135/9781849209953

Rosch, E. H., \& Mervis, C. B. (1975). Family resemblances: Studies in the internal structure of categories. Cognitive Psychology, 74), 573-605. https://doi.org/10.1016/0010-0285(75)90024-9

Rose, S. B., \& Abdel Rahman, R. (2016). Cumulative semantic interference for associative relations in language production. Cognition, 152, 20-31. https://doi.org/10.1016/j.cognition.2016.03.013

Rose, S. B., \& Abdel Rahman, R. (2017). Semantic similarity promotes interference in the continuous naming paradigm: Behavioural and electrophysiological evidence. Language, Cognition and Neuroscience, 32(1), 55-68. https://doi.org/10.1080/23273798.2016.1212081

Rose, S. B., Aristei, S., Melinger, A., \& Abdel Rahman, R. (2019). The closer they are, the more they interfere: Semantic similarity of word distractors increases competition in language production. Journal of Experimental Psychology: Learning, Memory, and Cognition, 45(4), 753-763. https://doi.org/10.1037/xIm0000592

Rossion, B., \& Pourtois, G. (2004). Revisiting Snodgrass and Vanderwart's object pictorial set: The role of surface detail in basic-level object recognition. Perception, 33(2), 217-236. https://doi.org/10.1068/p5117

RStudio Team. (2019). RStudio: Integrated development for R (1.2.5033). RStudio, Inc. http://www.rstudio.com

Snodgrass, J. G., \& Vanderwart, M. (1980). A standardized set of 260 pictures: Norms for name agreement, image agreement, familiarity, and visual complexity. Journal of Experimental Psychology: Human Learning and Memory, 6(2), 174-215. https://doi.org/10.1037/02787393.6.2.174

Taylor, K. I., Devereux, B. J., Acres, K., Randall, B., \& Tyler, L. K. (2012). Contrasting effects of featurebased statistics on the categorisation and basic-level identification of visual objects. Cognition, 
122(3), 363-374. https://doi.org/10.1016/j.cognition.2011.11.001

Taylor, K. I., Moss, H., Randall, B., \& Tyler, L. K. (2004). The interplay between distinctiveness and intercorrelation in the automatic activation of word meaning. Proceedings of the 45th Annual Meeting of the Psychonomic Society, 9, 109.

Toglia, M. P., \& Battig, W. F. (1978). Handbook of semantic word norms. Lawrence Erlbaum Associates.

Tyler, L. K., \& Moss, H. E. (2001). Towards a distributed account of conceptual knowledge. Trends in Cognitive Sciences, 5(6), 244-252. https://doi.org/10.1016/S1364-6613(00)01651-X

Tyler, L. K., Moss, H. E., Durrant-Peatfield, M. R., \& Levy, J. P. (2000). Conceptual structure and the structure of concepts: A distributed account of category-specific deficits. Brain and Language, 75(2), 195-231. https://doi.org/10.1006/brln.2000.2353

van Heuven, W. J. B., Mandera, P., Keuleers, E., \& Brysbaert, M. (2014). SUBTLEX-UK: A new and improved word frequency database for British English. Quarterly Journal of Experimental Psychology, 67(6), 1176-1190. https://doi.org/10.1080/17470218.2013.850521

Vieth, H. E., McMahon, K. L., \& de Zubicaray, G. I. (2014). Feature overlap slows lexical selection: Evidence from the picture-word interference paradigm. Quarterly Journal of Experimental Psychology, 67(12), 2325-2339. https://doi.org/10.1080/17470218.2014.923922

Vinson, D. P., \& Vigliocco, G. (2008). Semantic feature production norms for a large set of objects and events. Behavior Research Methods, 4O(1), 183-190. https://doi.org/10.3758/BRM.40.1.183

Vitkovitch, M., \& Humphreys, G. W. (1991). Perseverant responding in speeded naming of pictures: It's in the links. Journal of Experimental Psychology: Learning, Memory, and Cognition, 17(4), 664680. https://doi.org/10.1037/0278-7393.17.4.664

Woollams, A. M. (2012). Apples are not the only fruit: The effects of concept typicality on semantic representation in the anterior temporal lobe. Frontiers in Human Neuroscience, 6, 1-9. https://doi.org/10.3389/fnhum.2012.00085 


\section{Appendices}

\section{Appendix A}

Composition of the six experimental lists

\section{Table A1}

Composition of the different experimental lists

\begin{tabular}{|c|c|c|c|c|}
\hline & Position 1 & Position 2 & Position 3 & Position 4 \\
\hline List 1 & $\begin{array}{l}\text { Block 1- } \\
\text { Pseudorand. } 1\end{array}$ & $\begin{array}{l}\text { Block 2- } \\
\text { Pseudorand. } 1\end{array}$ & $\begin{array}{l}\text { Block } 3 \text { - } \\
\text { Pseudorand. } 1\end{array}$ & $\begin{array}{l}\text { Block } 4- \\
\text { Pseudorand. } 1\end{array}$ \\
\hline List 2 & $\begin{array}{l}\text { Block } 1 \text { - } \\
\text { Pseudorand. } 1\end{array}$ & $\begin{array}{l}\text { Block } 2 \text { - } \\
\text { Pseudorand. } 1\end{array}$ & $\begin{array}{l}\text { Block } 4 \text { - } \\
\text { Pseudorand. } 1\end{array}$ & $\begin{array}{l}\text { Block } 3 \text { - } \\
\text { Pseudorand. } 1\end{array}$ \\
\hline List 3 & $\begin{array}{l}\text { Block } 1 \text { - } \\
\text { Pseudorand. } 2\end{array}$ & $\begin{array}{l}\text { Block } 3 \text { - } \\
\text { Pseudorand. } 2\end{array}$ & $\begin{array}{l}\text { Block } 4 \text { - } \\
\text { Pseudorand. } 2\end{array}$ & $\begin{array}{l}\text { Block } 2 \text { - } \\
\text { Pseudorand. } 2\end{array}$ \\
\hline List 4 & $\begin{array}{l}\text { Block } 1 \text { - } \\
\text { Pseudorand. } 2\end{array}$ & $\begin{array}{l}\text { Block } 3 \text { - } \\
\text { Pseudorand. } 2\end{array}$ & $\begin{array}{l}\text { Block } 2 \text { - } \\
\text { Pseudorand. } 2\end{array}$ & $\begin{array}{l}\text { Block } 4 \text { - } \\
\text { Pseudorand. } 2\end{array}$ \\
\hline List 5 & $\begin{array}{l}\text { Block } 1 \text { - } \\
\text { Pseudorand. } 3\end{array}$ & $\begin{array}{l}\text { Block } 4 \text { - } \\
\text { Pseudorand. } 3\end{array}$ & $\begin{array}{l}\text { Block } 2 \text { - } \\
\text { Pseudorand. } 3\end{array}$ & $\begin{array}{l}\text { Block } 3 \text { - } \\
\text { Pseudorand. } 3\end{array}$ \\
\hline List 6 & $\begin{array}{l}\text { Block } 1 \text { - } \\
\text { Pseudorand. } 3\end{array}$ & $\begin{array}{l}\text { Block } 4 \text { - } \\
\text { Pseudorand. } 3\end{array}$ & $\begin{array}{l}\text { Block } 3 \text { - } \\
\text { Pseudorand. } 3\end{array}$ & $\begin{array}{l}\text { Block } 2 \text { - } \\
\text { Pseudorand. } 3\end{array}$ \\
\hline
\end{tabular}

Note. Pseudorand. = Pseudorandomisation 


\section{Appendix B}

Detailed description of calculation of semantic variables based on information given in McRae et al. (2005)

\section{Number of semantic features}

Number of semantic features was the total number of semantic features produced by at least 5 out of 30 participants when generating features for the concept. Taxonomic features were excluded from this calculation and the calculation of all other semantic variables. This measure was directly retrieved from McRae et al. (2005).

\section{Intercorrelational density}

Intercorrelational density was calculated based on Pearson's product moment correlations between the pairs of features in McRae et al.'s (2005) concept-feature matrix (cells were filled with the production frequency of the particular feature for a concept). Features that appeared in less than three concepts were excluded to avoid spurious correlations.

Feature pairs were counted as significantly correlated if the two features shared at least $6.5 \%$ of their variance $\left(r^{2}\right)$. From this information, intercorrelational density of a concept was calculated as the sum of the shared variance of its significantly correlated feature pairs. This measure was directly retrieved from the database by McRae et al. (2005).

\section{Number of near semantic neighbours}

A near semantic neighbour was defined as a word that had feature overlap of at least .4 (cosine similarity between feature production frequency vectors in the database by McRae et al. (2005)) with the target word (following Mirman, 2011). The instances of near semantic neighbours were added for each concept to determine the number of near semantic neighbours.

\section{Semantic similarity}

Semantic similarity was the average similarity of the target word's feature production frequency vector and the feature vectors of all other concepts from the McRae et al. (2005) database (following Mirman \& Magnuson, 2008). 


\section{Typicality}

Typicality was calculated in a similar way to Rosch and Mervis' (1975) family resemblance score. First, the features of an item were weighted based on category affiliations. Each feature of an item was attributed with the number of other items in the same semantic category that were credited with that particular feature.

This value was then divided by the number of items in the semantic category, which results in a proportion. Such a division was not part of the original measure by Rosch and Mervis (1975), who used raw values to determine the family resemblance score; however, we decided for this step because the number of items in each semantic category was not uniform in our data, as opposed to Rosch and Mervis.

Subsequently, we amended the original family resemblance score calculations further by multiplying this value for each feature by its production frequency (number of participants who produced that feature for the target word when generating the feature norms) to account for feature importance (e.g., Garrard et al., 2005). As a final step, the feature weights of all features of an item were summed.

We considered four different ways to operationalise typicality: 1) taking production frequency of individual features into account or not, and 2) using either raw values or proportions based on the number of items in a particular semantic category. The four versions of the typicality measure were highly correlated with one another, however, the measure as described above (using proportions based on the number of items in a particular semantic category and taking production frequencies of individual features into account) correlated least strongly with the other semantic variables.

\section{Distinctiveness}

Distinctiveness of each feature of an item was calculated as the inverse of the number of concepts in which that feature occurred across the database. To determine conceptual distinctiveness, this feature distinctiveness was then averaged across the features of a concept. This measure was also directly retrieved from McRae et al. (2005). 


\section{Appendix C}

Comparison of semantic variables in the full McRae et al. (2005) database and the 297 items included in the experimental investigation in Study 2

The feature database by McRae et al. (2005) contains 541 concepts, however, here we only used a subset of these items ( $n=297)$ with high name agreement in Australian English, as identified in the norming study presented in Study 1. Importantly, and as shown in Table C1, the items selected for the experimental investigation in Study 2 were a good representation of the items of the full McRae et al. set as semantic variables were largely comparable for the selected items and the whole database. This suggests that the selection of high name agreement items in Australian English and the resulting reduction of the stimuli compared to the full McRae et al. database did most likely not affect the comparability of the findings of Study 2 to previous work that used the full McRae et al. database (i.e., Rabovsky et al., 2016).

\section{Table C1}

Descriptive statistics for the semantic variables included in Study 2 and the full McRae et al. (2005) database

\begin{tabular}{|c|c|c|c|c|c|c|}
\hline \multirow[b]{2}{*}{ Semantic variable } & \multicolumn{3}{|c|}{$\begin{array}{c}\text { Study } 2: \\
n=297 \text { items }\end{array}$} & \multicolumn{3}{|c|}{$\begin{array}{l}\text { McRae et al. (2005) full database: } \\
\qquad n=541 \text { items }\end{array}$} \\
\hline & Mean & $S D$ & Range & Mean & $S D$ & Range \\
\hline $\begin{array}{r}\text { Number of semantic } \\
\text { features }\end{array}$ & 12.71 & 2.99 & $6-20$ & 12.07 & 3.21 & $5-21$ \\
\hline Intercorrelational density & 153.40 & 172.19 & $0.00-1296.22$ & 175.06 & 205.66 & $0.00-1419.41$ \\
\hline Semantic similarity & 0.04 & 0.02 & $0.00-0.09$ & 0.04 & 0.02 & $0.00-0.09$ \\
\hline $\begin{array}{r}\text { Number of near semantic } \\
\text { neighbours }\end{array}$ & 6.14 & 7.55 & $0-38$ & 7.14 & 8.64 & $0-40$ \\
\hline Typicality & 32.64 & 16.14 & $4.22-91.25$ & 32.47 & 16.85 & $4.22-95.44$ \\
\hline Distinctiveness & 0.37 & 0.16 & $0.04-0.80$ & 0.35 & 0.17 & $0.03-0.80$ \\
\hline
\end{tabular}




\section{Appendix D}

Detailed response coding

\section{Table D1}

Response coding examples

\begin{tabular}{|c|c|c|c|c|}
\hline Target word & Response & Response type & RT & Accuracy \\
\hline Sofa & Sofa & Correct & RT & 1 \\
\hline Sofa & $\begin{array}{l}\text { A sofa } \\
\text { (determiner) }\end{array}$ & Correct with determiner & -1 & 1 \\
\hline Sofa & S sofa & $\begin{array}{l}\text { Disfluency on the initial } \\
\text { phoneme of target word }\end{array}$ & -1 & 1 \\
\hline Sofa & Erm sofa & Hesitation & -1 & 1 \\
\hline Sofa & Red sofa & Elaboration & -1 & 1 \\
\hline Sofa & Couch & $\begin{array}{l}\text { Synonym or } \\
\text { acceptable alternative }\end{array}$ & -1 & NA \\
\hline Submarine & Sub & Abbreviation & -1 & NA \\
\hline Bed & $\begin{array}{l}\text { Emu (previous } \\
\text { item: emu).. Bed }\end{array}$ & Previous & -1 & NA \\
\hline Squid & $\begin{array}{l}\text { O squid or } \\
\text { Oct squid }\end{array}$ & Disfluency with self-correction & -1 & 0 \\
\hline Sofa & So & Incomplete response & -1 & 0 \\
\hline Sofa & Chair & Error & -1 & 0 \\
\hline Sofa & I don't know & Omission & -1 & 0 \\
\hline
\end{tabular}

Note. $\mathrm{RT}=$ Response time, NA = not analysed; responses with RT -1 were excluded from the naming latency analyses. 


\section{Appendix E}

Visualisations of findings of Analyses $1 \mathrm{~A}$ and $1 \mathrm{~B}$

The findings of Analysis $1 \mathrm{~A}$ are graphically displayed in Figure E1, with Model 1A.1 in Panel A and Model 1A.2 in Panel B. Figure E2 displays the findings of Analysis 1B, with Model 1B.1 in Panel A and Model 1B.2 in Panel B. Red lines indicate a facilitatory effect of a variable with speeding of naming latency or higher naming accuracy as that variable increases in value, while blue lines indicate an inhibitory effect with slowing of naming latency or reduced naming accuracy as that variable increases in value. Confidence intervals that cross the black zero line are indicative of non-significant effects.

\section{Figure E1}

Analysis 1 A replicating Rabovsky et al. (2016): Fixed effects estimates with 95\% confidence interval of picture naming latency analysis (Panel A; Model 1A.1) and accuracy analysis (Panel B; Model 1A.2)

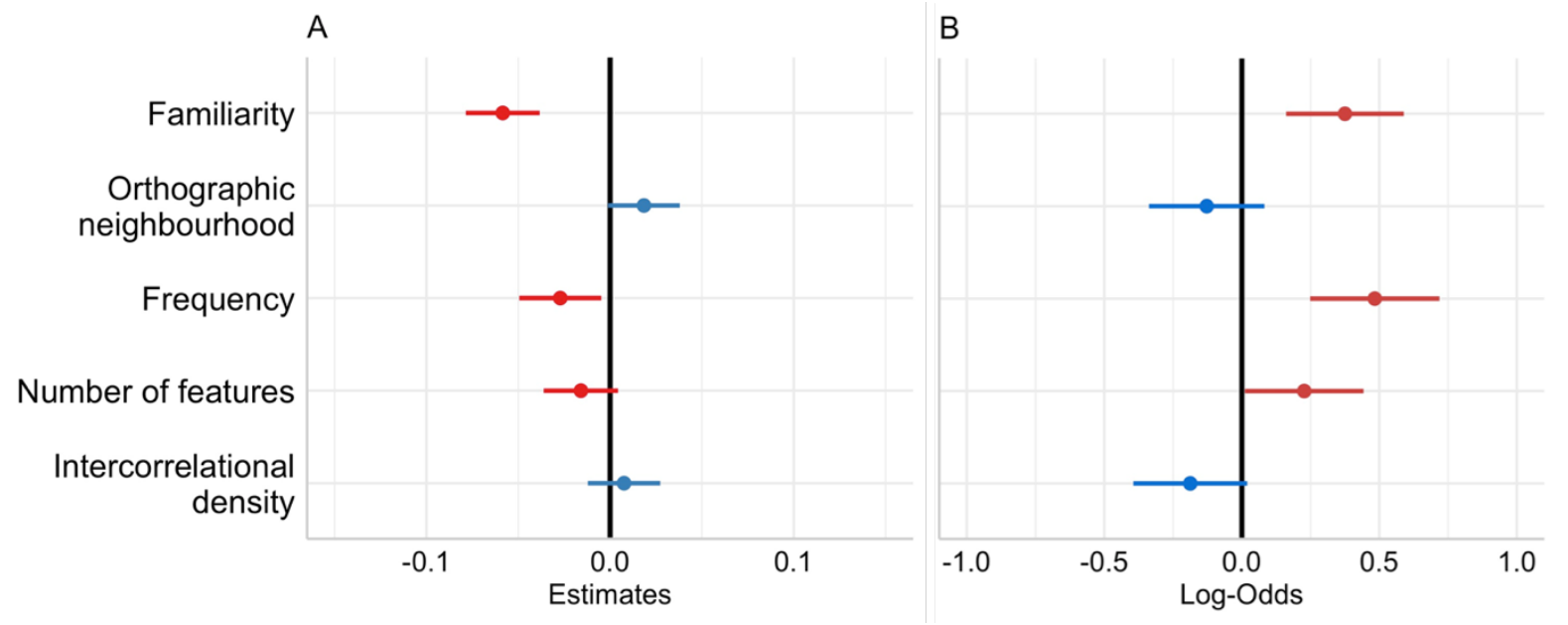

Note. Panel A shows the output of the naming latency analysis and Panel B the output of the naming accuracy analysis; red lines (to the left of centre for latency, and right for accuracy) indicate increased values of the variable lead to better performance, and blue lines (to the right of centre for latency, and left for accuracy) indicate worse performance. 


\section{Figure E2}

Analysis 1 B replicating Rabovsky et al. (2016) taking more psycholinguistic control variables into account: Fixed effects estimates with 95\% confidence interval of picture naming latency analysis (Panel A; Model 1B.1) and accuracy analysis (Panel B; Model 1B.2)

A

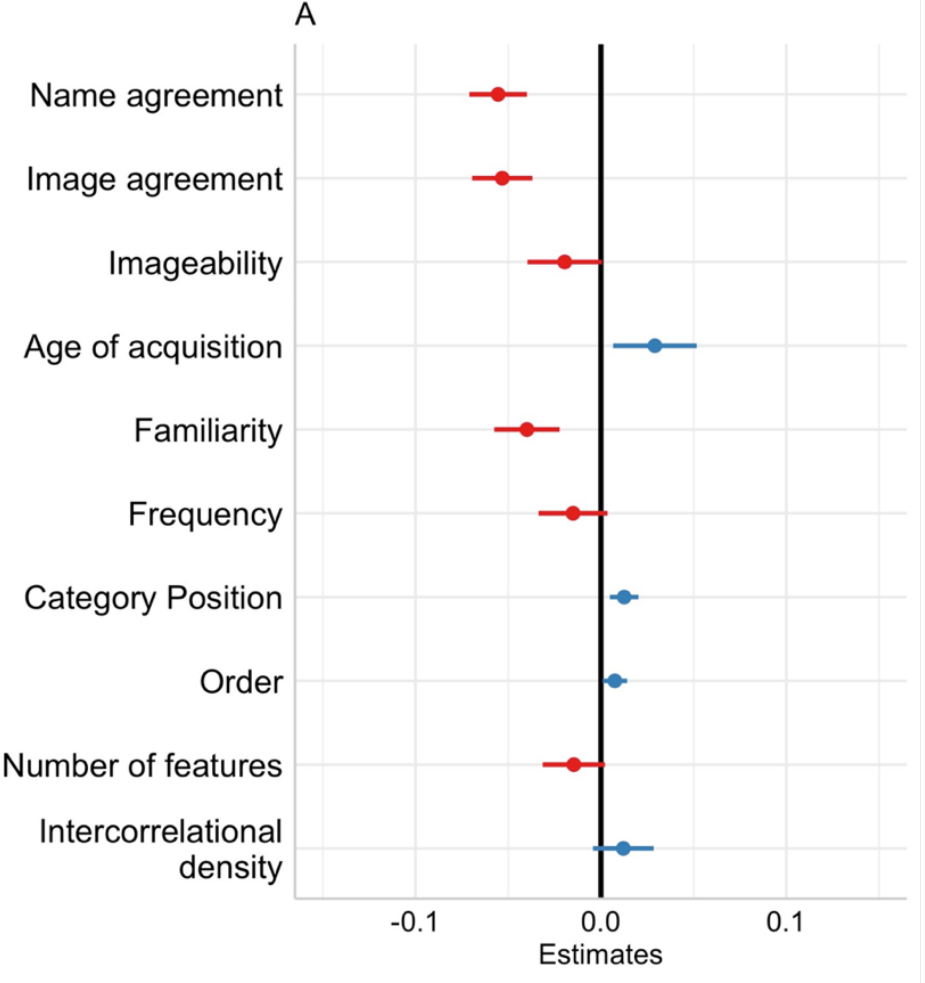

B

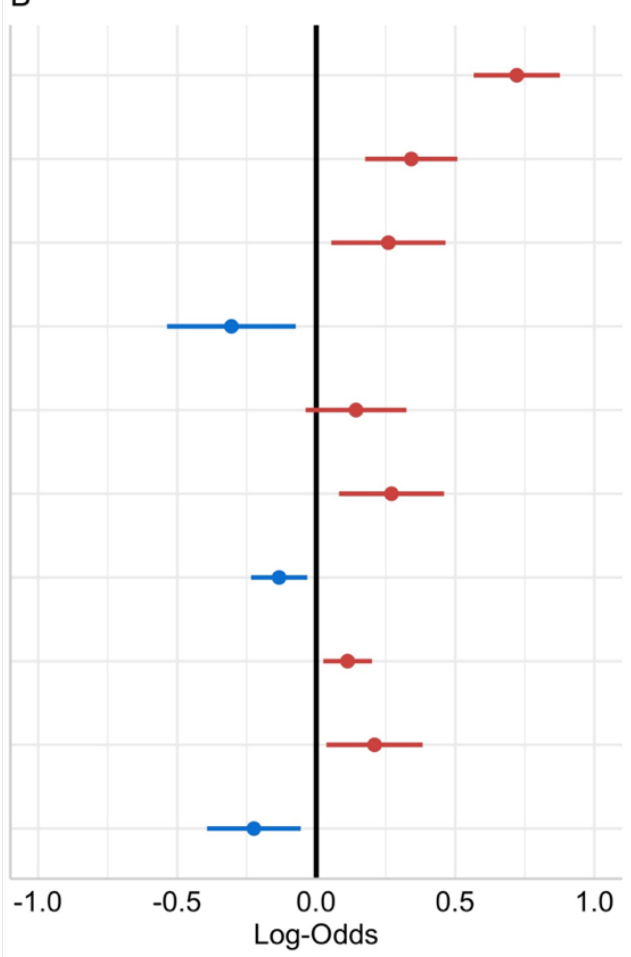

Note. Panel A shows the output of the naming latency analysis and Panel B the output of the naming accuracy analysis; red lines (to the left of centre for latency, and right for accuracy) indicate increased values of the variable lead to better performance, and blue lines (to the right of centre for latency, and left for accuracy) indicate worse performance. 


\section{Appendix F}

Post-hoc analyses of distinctiveness in living things: Testing the prediction of the Conceptual Structure Account

The Conceptual Structure Account predicts a disadvantage for distinctive features of living things. We therefore tested the explanatory strength of this account for our inhibitory effect of distinctiveness on naming latency and for naming accuracy.

We coded items of the semantic categories birds, fruit, invertebrate, land animal, plant, sea creature, and vegetable as living $(n=100)$ and all other items were coded as non-living.

The structure of the linear mixed effect model used for this exploratory analysis included the same fixed effects as the model of Analysis 2.1 with an additional interaction between distinctiveness and animacy. A random by-participant slope for number of near semantic neighbours was included.

The interaction between distinctiveness and animacy was significant $(\beta=-0.06, \mathrm{SE}=0.02, t=-$ 2.69, $p=.008$ ) and is displayed in Figure E1. While words of the living category were unaffected by distinctiveness, the inhibitory main effect of distinctiveness in Analysis 2 seemed to be driven by the items of the non-living category. This finding allows us to exclude the Conceptual Structure Account as an appropriate framework for the finding of this experiment.

\section{Figure E1}

Interaction between distinctiveness and animacy

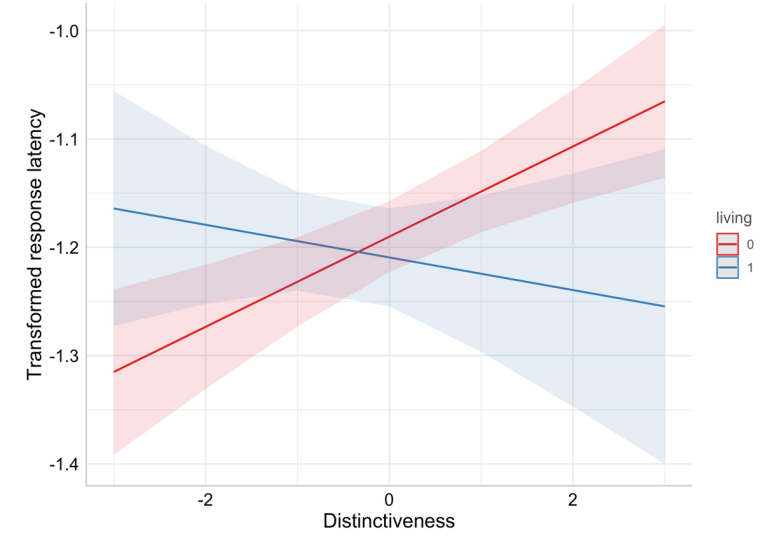


For naming accuracy, the generalised linear mixed effect model included the same fixed effects as Analysis 2.2 with the additional interaction between distinctiveness and animacy and a random by-participant slope for number of near semantic neighbours. The interaction between distinctiveness and animacy was non-significant $(\beta=0.10, \mathrm{SE}=0.22, z=0.45, p=.642)$. 